\title{
Epigenetic driver mutations in ARID1A shape cancer immune phenotype and immunotherapy
}

\author{
Jing Li, ${ }^{1,2}$ Weichao Wang, ${ }^{1,2}$ Yajia Zhang, ${ }^{3}$ Marcin Cieślik, ${ }^{3,4,5}$ Jipeng Guo, ${ }^{1,2}$ Mengyao Tan, ${ }^{3}$ Michael D. Green, ${ }^{1,2,6}$ Weimin Wang, ${ }^{1,2}$ \\ Heng Lin, ${ }^{1,2}$ Wei Li, ${ }^{1,2}$ Shuang Wei, ${ }^{1,2}$ Jiajia Zhou, ${ }^{1,2}$ Gaopeng Li, ${ }^{1,2}$ Xiaojun Jing, ${ }^{3}$ Linda Vatan, ${ }^{1,2}$ Lili Zhao, ${ }^{7}$ Benjamin Bitler, ${ }^{8}$ \\ Rugang Zhang, ${ }^{8}$ Kathleen R. Cho, ${ }^{3,5}$ Yali Dou, ${ }^{3,5}$ Ilona Kryczek, ${ }^{1,2}$ Timothy A. Chan, ${ }^{9}$ David Huntsman, ${ }^{10,11}$ \\ Arul M. Chinnaiyan, ${ }^{3,6,12,13,14}$ and Weiping Zou, ${ }^{1,2,3,5,15}$

\begin{abstract}
'Department of Surgery, University of Michigan School of Medicine, Ann Arbor, Michigan, USA. ${ }^{2}$ Center of Excellence for Cancer Immunology and Immunotherapy, University of Michigan Rogel Cancer Center, University of Michigan School of Medicine, Ann Arbor, Michigan, USA. ${ }^{3}$ Department of Pathology, ${ }^{4}$ Department of Computational Medicine and Bioinformatics, ${ }^{5}$ University of Michigan Rogel Cancer Center, and 'Department of Radiation Oncology, University of Michigan School of Medicine, Ann Arbor, Michigan, USA. Department of Biostatistics, University of Michigan, Ann Arbor, Michigan, USA. ${ }^{8}$ Cene Expression and Regulation Program, The Wistar Institute, Philadelphia, Pennsylvania, USA. ${ }^{9}$ Department of Radiation Oncology, Memorial Sloan Kettering Cancer Center, New York, New York, USA. ${ }^{10}$ Department of Molecular Oncology, British Columbia Cancer, Vancouver, British Columbia, Canada. "Department of Pathology and Laboratory Medicine, University of British Columbia, Vancouver, British Columbia, Canada. ${ }^{2}$ Department of Urology, ${ }^{13}$ Michigan Center for Translational Pathology, ${ }^{14}$ Howard Hughes Medical Institute, and ${ }^{15}$ Craduate Program in Immunology and Graduate Program in Cancer
\end{abstract} \\ Biology, University of Michigan School of Medicine, Ann Arbor, Michigan, USA.
}

\begin{abstract}
Whether mutations in cancer driver genes directly affect cancer immune phenotype and T cell immunity remains a standing question. ARID1A is a core member of the polymorphic BRG/BRM-associated factor chromatin remodeling complex. ARID1A mutations occur in human cancers and drive cancer development. Here, we studied the molecular, cellular, and clinical impact of ARID1A aberrations on cancer immunity. We demonstrated that ARID1A aberrations resulted in limited chromatin accessibility to IFN-responsive genes, impaired IFN gene expression, anemic T cell tumor infiltration, poor tumor immunity, and shortened host survival in many human cancer histologies and in murine cancer models. Impaired IFN signaling was associated with poor immunotherapy response. Mechanistically, ARID1A interacted with EZH2 via its carboxyl terminal and antagonized EZH2-mediated IFN responsiveness. Thus, the interaction between ARID1A and EZH2 defines cancer IFN responsiveness and immune evasion. Our work indicates that cancer epigenetic driver mutations can shape cancer immune phenotype and immunotherapy.
\end{abstract}

\section{Introduction}

Tumor-infiltrating $\mathrm{T}$ cells are positively associated with patient survival in many types of human cancer (1-3). Type I and type II IFN gene signatures correlate with clinical responses to cancer immunotherapy (4-7), biological antibody therapy, chemotherapy, and radiation therapy (8-10). Given the role of key genetic mutations in cancer initiation and progression, it has long been argued that driver mutation(s) may drive the cancer immune phenotype and immune tolerance in patients with cancer. However, there is insufficient direct evidence in patients backing a causal link between cancer $\mathrm{T}$ cell immunity and multiple, frequent cancer driver genetic mutations, including MYC, APC, TP53, and KRAS. Notably, it has been reported that Myc amplification may induce an immunosuppressive tumor microenvironment via CCL9 and IL-23, which recruits macrophages and limits NK cell tumor trafficking in a mouse model of lung adenoma (11). Along this line,

Authorship note: JL and Weichao Wang contributed equally to this work. Conflict of interest: WZ has served as a scientific advisor for Cstone, Oncopia, and Hengenix.

Copyright: (C) 2020, American Society for Clinical Investigation.

Submitted: October 21, 2019; Accepted: January 30, 2020; Published: April 20, 2020.

Reference information: / Clin Invest. 2020;130(5):2712-2726.

https://doi.org/10.1172/JCl134402.
Myc amplification may transcriptionally regulate immune inhibition-associated molecules such as CD47 and PD-L1 in a mouse model of T cell acute lymphoblastic leukemia (12). In addition, $\mathrm{Kras}^{\mathrm{Gi2D}}$ can cause an increase in expression of the inflammatory cytokine GM-CSF in a mouse model of pancreatic neoplasia (13). Loss of P53 function activates the JAK2-STAT3 signaling pathway and promotes pancreatic tumor growth (14), and mutant P53 prolongs NF-kB activation and increases colorectal tumor incidence in a mouse model (15). These studies reveal a general mechanistic connection between cancer driver mutation-related inflammation and cancer progression in different model systems. However, the link among cancer driver gene mutations, $\mathrm{T}$ cell immunity, and immunotherapy response has not been established in patients with cancer. Thus, we have attempted to explore whether cancer genetic driver mutation(s) are capable of directly driving cancer immune phenotype, contributing to IFN signature and T cell immunity, and affecting immunotherapy response in models and in patients with cancer. Given that the most recurrent cancer driver genetic mutations, including MYC, APC, TP53, and KRAS, have been examined, in this work we focused our studies on the cancer driver epigenetic mutations such as ARID1A mutations (16-19).

ARID1A belongs to the SWI/SNF complex and is a core member of the ATP-dependent polymorphic BRG/BRM-associated factor (BAF) chromatin remodeling unit. SWI/SNF complex mutations 
are found in $20 \%$ of all types of human cancer $(19,20)$. ARID1A mutations occur in many types of human cancer, including ovarian clear cell carcinoma (OCCC) with a 50\% mutation rate (16-19). There are at least 29 components in the SWI/SNF complex. Mutations of different SWI/SNF components may have immunological and biological effects that are tumor type and context dependent (21). ARID1A has the highest mutation rate across all components in the SWI/SNF complex. In this work, we focus on ARID1A and its immunological impact on cancer immunity and immunotherapy.

Compared with the SWI/SNF complex, the polycomb repressive complex 2 (PRC2) has been relatively well studied in the context of cancer biology. Interestingly, it has been long speculated that there is genetic antagonism between the SWI/SNF complex and PRC2 (22-26). However, it is unknown whether the SWI/SNF complex and PRC2 biochemically, genetically, and biologically interact in the context of human cancers, and if so, whether this interaction plays a functional role in shaping cancer immune phenotype and T cell immunity. Enhancer of Zeste 2 PRC2 subunit (EZH2) is the catalytic subunit of PRC2 and functions as a methyltransferase of histone $\mathrm{H} 3$ on lysine 27 (H3K27). EZH2 generally mediates gene repression and plays an oncogenic role in a variety of cancer types. We have previously shown that EZH2 represses Th1-type chemokine (CXCL9 and CXCL10) expression and alters effector T cell tumor trafficking $(27,28)$. Thus, we hypothesized that there exists an active interaction between ARID1A and EZH2, this interaction affects $\mathrm{T}$ cell immunity, and ARID1A mutations functionally alter the interplay between EZH2 and ARID1A in tumors.

To test these hypotheses, we systematically studied the molecular, cellular, and clinical impact of ARID1A (mutations, expression levels, and copy number) on Th1-type chemokine expression, effector $\mathrm{T}$ cell tumor trafficking, IFN gene chromatin accessibility, and cancer immunity in OCCC, cutaneous melanoma, colon adenocarcinoma, and several other types of human cancer. Our data show that cancer epigenetic driver mutations such as ARIDIA mutations can shape tumor immune phenotype, $\mathrm{T}$ cell immunity, and the efficacy of cancer immunotherapy.

\section{Results}

ARID1A gene status correlates with cancer immune signature. Inactivating ARID1A mutations occur in $50 \%$ of OCCCs $(16,17)$. To explore a potential link between ARID1A mutations and the tumor immune responses in the human cancer microenvironment, we initially examined the relationship between ARID1A mutations and immune parameters in human OCCCs. We analyzed a published RNA-Seq data set of 18 OCCCs that was originally used to identify ARID1A mutations (16). Gene set enrichment analysis (GSEA) of this data set demonstrated significantly enriched pathways of Th1-type immune response, cytotoxic $\mathrm{T}$ cell response, and NK cell activation in ARID1A WT OCCCs as compared with ARID1A-mutated ovarian clear carcinomas (Figure 1, A-C, and Supplemental Table 1; supplemental material available online with this article; https://doi.org/10.1172/JCI134402DS1). In line with this analysis, WT ARID1A OCCCs expressed higher levels of type II IFN signature genes, including Th1-type chemokines, CXCL9, CXCL10, and CXCL11, as compared with ARID1Amutated cancers (Figure 1, D-F). However, ARID1A-mutated cancers expressed higher levels of CXCL8 (Supplemental Figure
1A) and similar levels of IL-6 (Supplemental Figure 1B) and IL-32 (Supplemental Figure 1C) as compared with WT ARID1A cancer, suggesting that ARID1A mutations do not induce global repression of chemokine and cytokine expression. We also found enriched tumor-infiltrating $\mathrm{T}$ cells as shown by high levels of $\mathrm{T}$ cell receptor CDR3 reads (Figure 1G) and CD8 expression (Figure 1H) in WT ARID1A cancers as compared with those with mutant ARID1A. In addition, WT and mutated ARID1A ovarian clear carcinomas had comparable levels of FOXP3, a marker for $\mathrm{CD}_{4}{ }^{+}$Treg cells (Supplemental Figure 1D). The data suggest that ARID1A mutations do not uniformly affect immune cell subset tumor infiltration. We extended our analyses to ovarian cancer patients with metastatic disease (29). We detected higher levels of IRF1 expression in WT ARID1A tumors as compared with mutated ARID1A tumors (Supplemental Figure 1E), Th1-type chemokines (Supplemental Figure 1, F-H), and infiltrating $\mathrm{CD}^{+} \mathrm{T}$ cell and cytotoxic $\mathrm{T}$ cell effector genes, granzyme B, and perforin (Supplemental Figure 1, $\mathrm{I}-\mathrm{K}$ ) in metastatic ovarian carcinomas with WT ARID1A as compared with those with mutated ARID1A (29). Analysis of another independent data set on OCCC confirmed that ARID1A expression strongly correlated to CXCL10, CD8A, perforin, and IRF1 gene expression (Figure 1, I-L, and ref. 30). The data suggest that ARID1A positively regulates IFN gene signaling in human ovarian cancer, particularly OCCC.

In addition to ovarian cancer, we analyzed the correlation of ARID1A expression levels with immune signatures in melanoma patients (31). ARID1A gene expression levels positively correlated with the IFN signature genes, including CXCL9, CXCL10, CXCL11, and IRF1 (Figure 1, M-P). This correlation was not confounded by tumor purity, as ARID1A levels were not associated with tumor purity (Supplemental Figure 1L). When ARID1A somatic copy number was taken into account, we noticed a positive correlation between ARID1A copy number and $\mathrm{CD} 8^{+} \mathrm{T}$ cell infiltration in patients with melanoma (Supplemental Figure 1M and ref. 31). Thus, there is also a positive correlation between ARIDIA and IFN signature genes in human melanoma.

We extended our analyses from ovarian cancer and melanoma to the pan-cancer data sets from The Cancer Genome Atlas (TCGA) compendium. In line with our data on OCCC and melanoma, we found that expression levels of CXCL9, CXCL10, CXCL11, and CD8A were higher in patients with high ARID1A mRNA expression than patients with low ARID1A (Figure 1, Q-T, and ref. 32). Furthermore, a positive correlation between ARID1A gene expression and $\mathrm{CD} 8^{+} \mathrm{T}$ cell infiltration was observed across many cancer types (Supplemental Figure 1N). Collectively, the data strongly suggest that ARID1A positively regulates IFN and $\mathrm{T}$ cell signaling and correlates to a protective tumor immunity in patients with cancer.

ARID1A mutations impair IFN signaling pathways in cancer. Th1-type chemokines are key IFN-responsive genes and mediate effector $\mathrm{T}$ cell tumor trafficking. Based on our observations (Figure 1), we hypothesized that ARID1A is an epigenetic regulator of IFN signaling pathways in tumor cells. To test this hypothesis, using CRISPR/Cas9 genome editing technology, we generated ARID1A-knockout OCCC cells (AC17 and AC25) from parental ovarian clear cancer cells (OVCA429) (Figure 2A), and examined their responses to type II IFN (IFN- $\gamma$ ) stimulation. We observed 
A

GSEA analysis in ARID1A WT and MUT OCCC

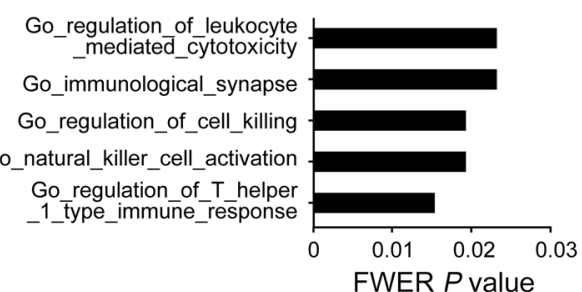

B Enrichment plot

GO_Regulation_of_T_Helper_1_Type

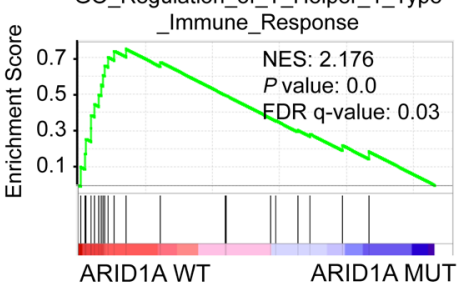

C

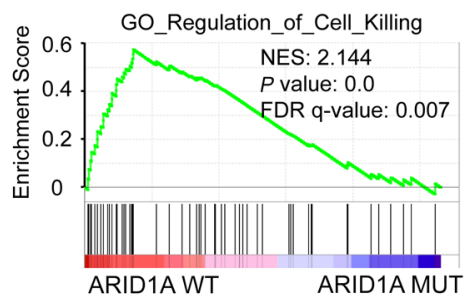

D

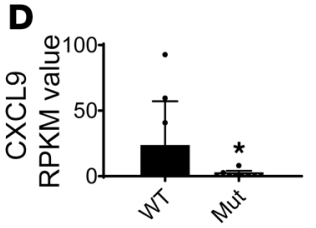

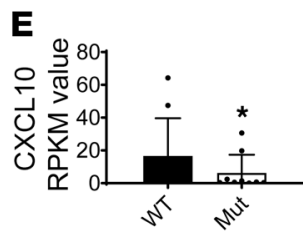

F

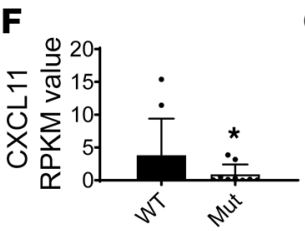

G

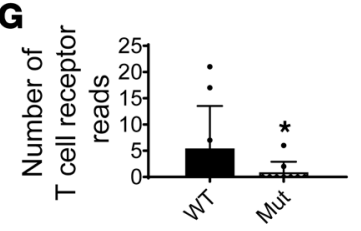

H

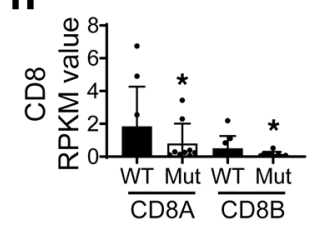

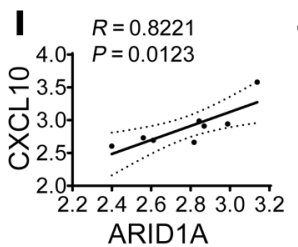
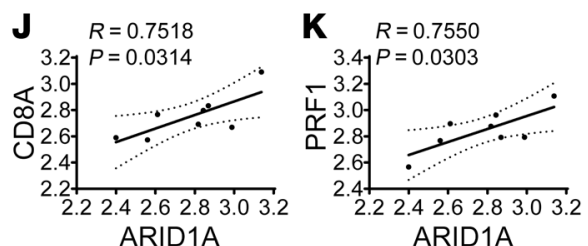

ARID1A

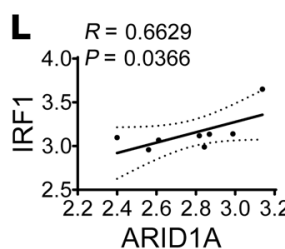

ARID1A
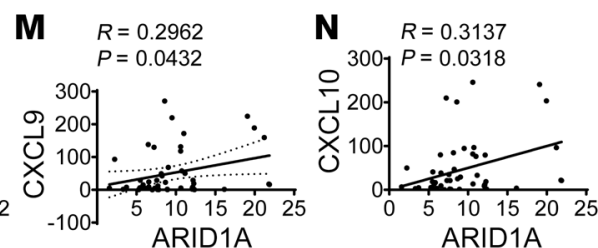
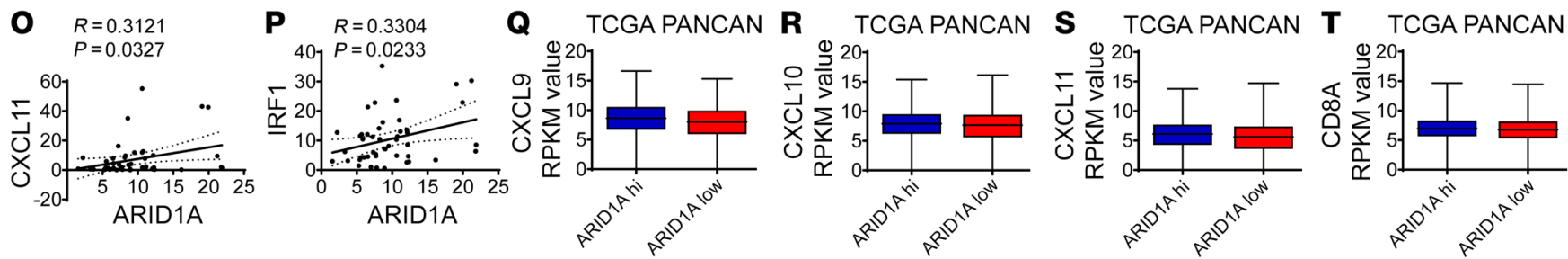

Figure 1. ARID1A gene status correlates with cancer immune signature. (A-H) Relationship between ARID1A mutations and immune signature genes. RNA-Seq was conducted in patients with OCCC. Nine patients with ARID1A mutations, 9 patients with WT ARID1A. (A) Top 5 GSEA pathways of transcriptome between WT and mutated ARID1A cancers are shown. (B) Th1-type immune response GSEA pathway is enriched in WT ARID1A OCCC patients, $\underline{Q}=$ 0.025529487. (C) Cytotoxic gene signatures were enriched in WT ARID1A OCCC patients, $\underline{Q}=0.007425$. (D-H) CXCL9 (D), CXCL10 (E), CXCL11 (F), TCR (G), and CD8 (H) RPKM values of represented transcripts are shown. ${ }^{*} P<0.05$. (I-L) ARID1A gene expression levels correlated with CXCL10 (I), CD8A (J), PRF1 (K), IRF1 (L) in 8 OCCC patients from Wu et al. study (30). (M-P) ARID1A gene expression levels correlated with CXCL9 (M), CXCL10 (N), CXCL11 (0), and IRF1 (P) gene expression levels in 47 WT ARID1A metastatic melanoma patients. (Q-T) CXCL9 (Q), CXCL10 (R), CXCL11 (S), CD8A (T) gene expression levels were higher in ARID1A high group, compared with ARID1A low group in TCGA pan-cancer (PANCAN) data set: 3838 patients in ARID1A high group, 3839 patients in ARID1A low group. For box-and-whisker plots in Q-T, the center line denotes the median value (50th percentile); the box contains the 25th to 75th percentiles of the data set. The whiskers mark the maximum and minimum values, ${ }^{*} P<0.001$.

that IFN- $\gamma$ stimulation resulted in comparable levels of STAT1 phosphorylation and IRF1 activation in ARID1A WT and knockout ovarian clear cancer cells (Figure 2A). However, ARID1A-knockout ovarian clear cancer cells expressed minimal levels of Th1type chemokine CXCL9 and CXCL10 mRNA as compared with their parental cells (Figure 2B). We included primary high-grade serous ovarian cancer cells (OC8) in our studies (33) and generated ARID1A-knockout cells from parental OC8 cells. Again, ARID1A-knockout OC8 cells expressed high levels of p-STAT1 and IRF1 (Supplemental Figure 2A) and low levels of CXCL9 and CXCL10 transcripts (Figure 2C) and proteins (Figure 2D) in response to IFN- $\gamma$ as compared with parental ARID1A WT cells. In order to examine the role of ARID1A in IFN- $\gamma$ responsiveness in different cancer histologies, we knocked down ARID1A in human DLD-1 colorectal cancer cells. In support of our observations in ovarian cancer, specific siRNA against ARID1A (Supplemental
Figure 2B) led to reduced CXCL9 and CXCL10 expression in human DLD-1 cells (Figure 2E). Specific shRNA against ARID1A also led to reduced CXCL9 and CXCL10 expression in the human primary colorectal cancer cells (Figure 2F and ref. 34). ARID1A deletion did not affect OCCC (OVCA429) and high-grade serous carcinoma (OC8) cell growth in vitro (Supplemental Figure 2, C and D). Thus, ARID1A affects the type II IFN signaling pathway in multiple human cancer histologies.

We next examined the responses of WT ARID1A parental ovarian clear cancer cells (OVCA429) and ARID1A-knockout ovarian clear cancer cells (AC17, AC25) to type I IFN. Again, ARID1A-knockout ovarian cancer cells expressed minimal levels of CXCL9 and CXCL10 as compared with ARID1A WT parental ovarian cancer cells in response to IFN- $\beta$ (Figure $2 \mathrm{G}$ and Supplemental Figure 2E). As a confirmation, our RNA-Seq data demonstrated poor type II (Figure 2H) and type I (Figure 2I) IFN signal- 


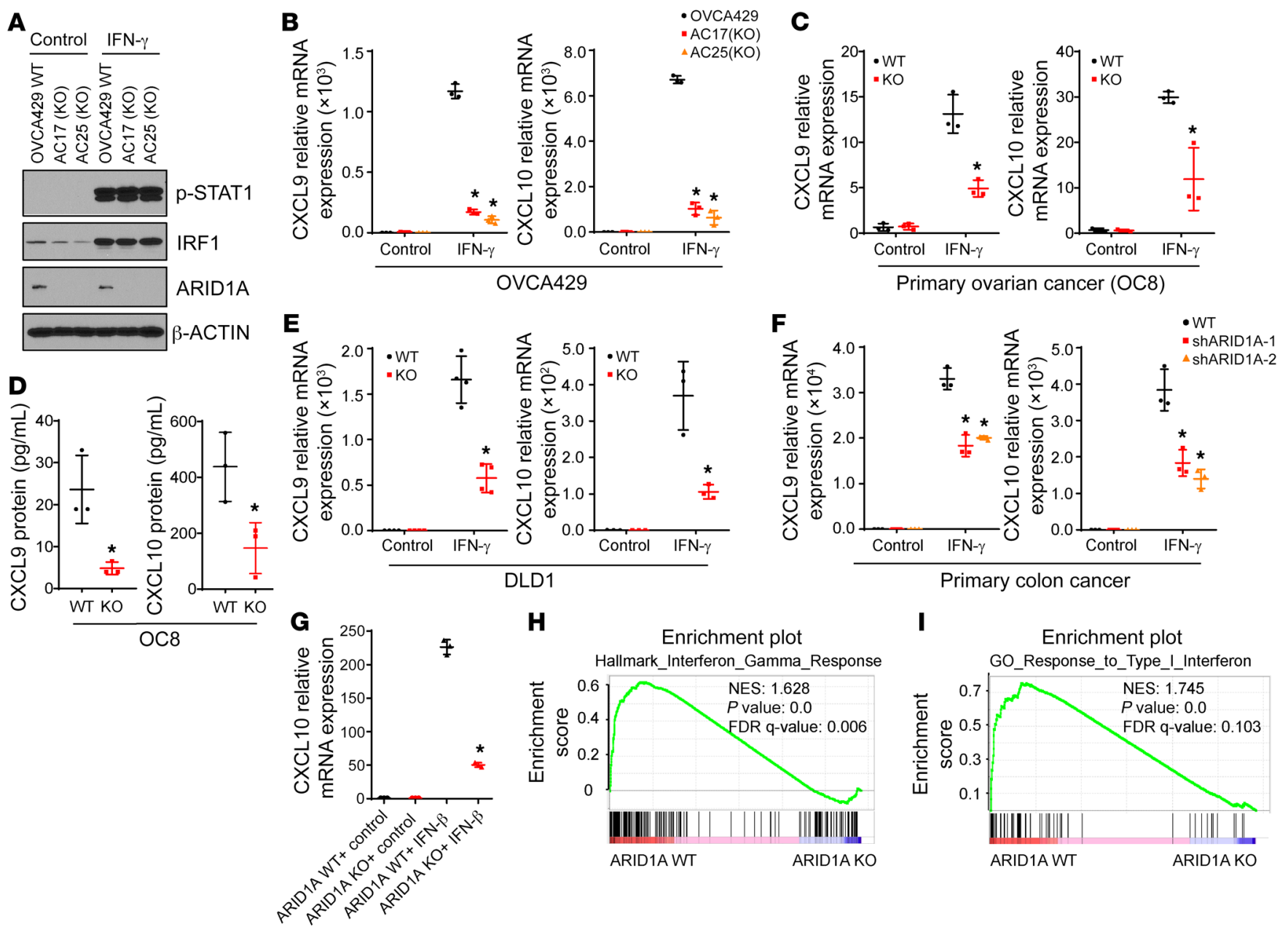

Figure 2. ARID1A mutations impair IFN signaling pathways in tumor. (A) Effects of ARID1A knockout on STAT1 activation and IRF1 induction. Two ARID1A-knockout OCCC clones (AC17 and AC25) were generated from the parental cell line OVCA429. Cells were treated with IFN- $\gamma$ for 24 hours. Relevant proteins were detected by Western blotting. One of 3 repeats is shown (uncut gels are in the online supplemental material). (B-F) Effect of ARID1A on IFN- $\gamma$-induced CXCL9 and CXCL10 expression in different types of human cancers (primer sequence is in Supplemental Table 8). Human ovarian clear cell cancer cell lines (B), primary serous ovarian cancer cells $(\mathbf{O C} 8)(\mathbf{C}$ and $\mathbf{D})$, colon cancer cell line DLD-1 (E), and primary colon cancer cells $(\mathbf{F})$ were treated with IFN- $\gamma$ for indicated hours. Chemokine expression was quantified by real-time PCR (B, C, E, and F) or ELISA (D). (Mean \pm SD, $\left.n=3-4,{ }^{*} P<0.05\right)$. (C) Effect of ARID1A on IFN- $\gamma$-induced CXCL10 expression. WT and knockout ARID1A ovarian clear cell cancer cells were treated with IFN- $\gamma$ for 8 hours. CXCL10 expression was quantified by real-time PCR. (Mean $\pm \mathrm{SD}, n=3,{ }^{*} P<0.05$ ). (H and I) Effect of ARID1A on type II (H) and type I (I) IFN gene signatures; WT and knockout ARID1A ovarian clear cell cancer cells were subjected to RNA-Seq. Based on the RNA-Seq data, GSEA was performed. ${ }^{*} P=0.00$ (H and I) FDR $\underline{Q}$ value $=0.0065(\mathbf{H})$, FDR $\underline{Q}$ value $=0.1(\mathbf{I})$.

ing pathways (Supplemental Table 2) in ARID1A-knockout ovarian cancer cells as compared with ARID1A WT parental ovarian cancer cells. In line with human studies, IFN signaling gene pathways were impaired in mouse colorectal cancer epithelial cells isolated from ARID1A genetically deficient mice as compared with mice with intact ARID1A alleles (ref. 35 and Supplemental Figure 2F). To determine whether ARID1A-mediated IFN signaling gene regulation is related to tumor cell genetic background, particularly defective DNA mismatch repair (MMR), we performed whole exome sequencing of DNA from OVCA429 and OC8 (MMR proficient) cells and DLD-1 (MMR deficient) cells. We found that OVCA429, OC8, and DLD-1 cells exhibited different mutation loads (Supplemental Figure 2, G and H, and Supplemental Tables 3 and 4). Thus, ARID1A-regulated IFN signaling does not appear to depend on MMR status and mutation load.
ARID1A regulates IFN- $\gamma$ signaling gene chromatin accessibility. Given that ARID1A is a core member of the BAF chromatin remodeling complex, we hypothesized that ARID1A dictated chromatin accessibility for IFN- $\gamma$-induced transcription. We used the assay for transposase accessible chromatin with high-throughput sequencing (ATAC-Seq), to assess and compare chromatin accessibility changes in ARID1A-deficient or WT human OCCC cells, with or without IFN- $\gamma$ treatment. After IFN- $\gamma$ treatment, a substantially larger number of genomic sites became more accessible in ARID1A-proficient cells than in deficient cells (Figure 3A), which was consistent with the RNA-Seq data (Figure 1 and Figure 2, $\mathrm{H}$ and I). We categorized sites with increased accessibility after IFN- $\gamma$ into 3 clusters (Figure 3B): those commonly observed in both ARID1A WT and knockout cells (cluster II) and those specific to either genetic background (WT cluster I and knockout III, respec- 


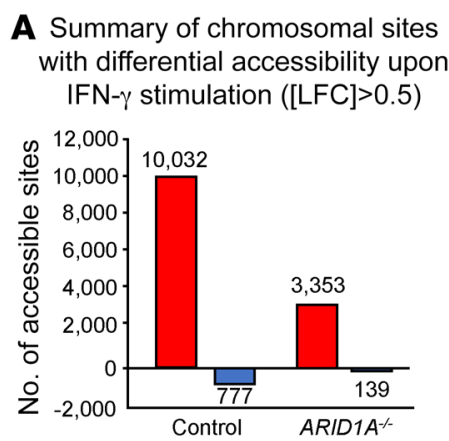

D

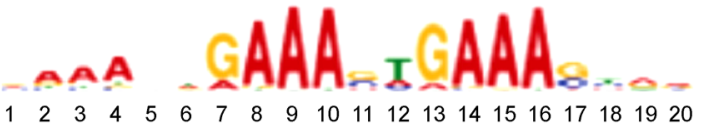

B Three clusters of more accessible sites following IFN- $\gamma$ treatment

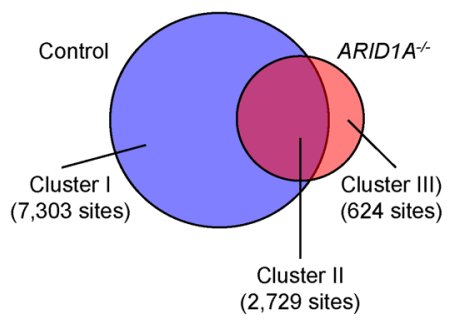

C Control Control + IFN- $\gamma$
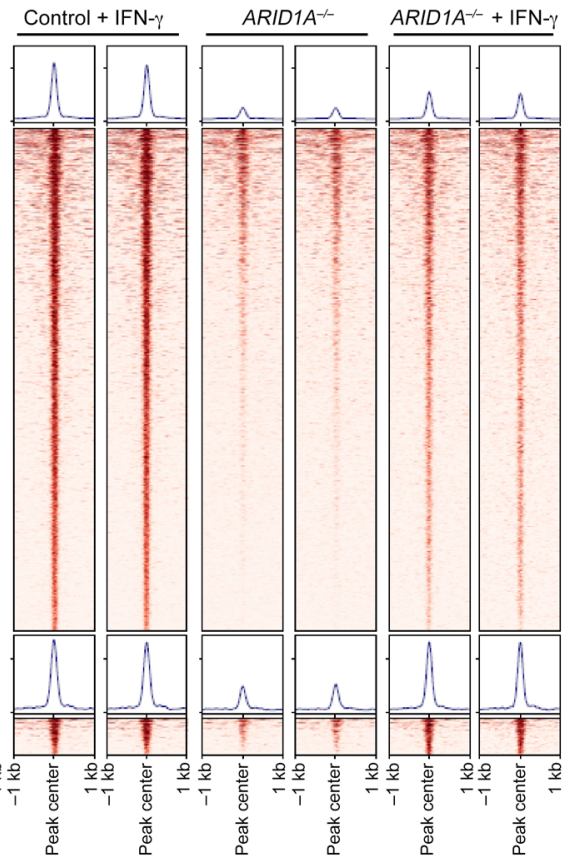

$\begin{array}{llllll}0.0 & 0.2 & 0.4 & 0.6 & 0.8 & 1.0\end{array}$

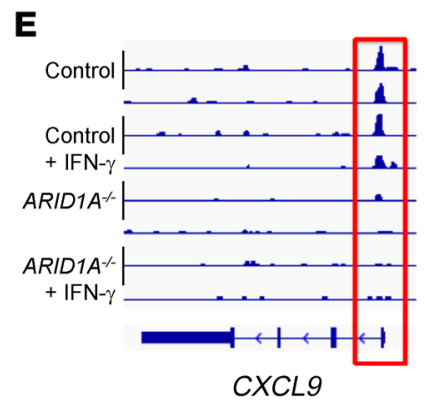

E

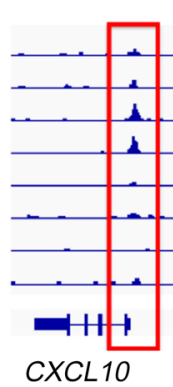

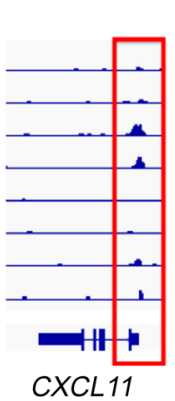

Fromoter accessibility of IFN- $\gamma$ ARID1A affected genes

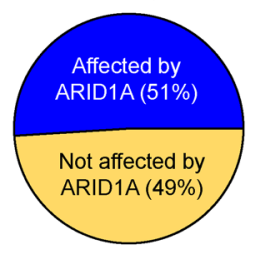

G

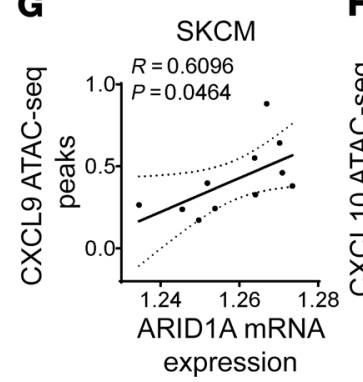

H

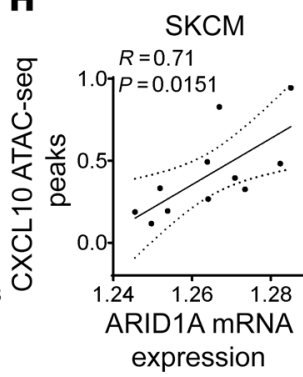

Figure 3. ARID1A regulates IFN- $\gamma$-signaling gene chromatin accessibility. (A and B) Genome-wide analysis (A) and Venn diagram (B) showing differentially accessible chromatin sites ( $|\mathrm{LFC}|>0.5$ ) after IFN- $\gamma$ stimulation in ARID1A-proficient (WT) and ARID1A-deficient (KO) OVCA-429 cells. (C) Chromatin accessibility heatmaps of ARID1A-proficient (WT) and ARID1A-deficient (KO) OVCA-429 cells. The heatmaps demonstrated the chromatin sites in cluster I (top) and cluster III (bottom). Aggregated peak intensity within $1 \mathrm{~kb}$ center of chromatin regions with differential accessibility is shown. (D) IRF2-binding motif was among the most significantly enriched motifs in clusters I, II, and III. (E) Examples of IFN- $\gamma$-responsive sites with less accessibilities in ARID1Adeficient (KO) OVCA-429 cells. The graph shows accessible sites near CXCL9, CXCL10, and CXCL11. (F) Pie chart illustrating accessibility changes of chromosomal sites adjacent to promoters (within $5 \mathrm{~kb}$ ) of IFN- $\gamma$-responsive and ARID1A-affected genes. Blue: Promoters with differentially accessible sites following ARID1A loss. Yellow: Promoters without significant changed sites following ARID1A loss. (G and $\mathbf{H})$ Correlation between ARID1A expression and average chromatin accessibility peaks near CXCL9 gene (221 peaks) (C) and CXCL10 (H) (219 peaks). Each dot represents an individual donor. ARID1A gene expression is log transformed. Eleven patients with WT skin cutaneous melanoma. $P=0.0464$ (G), $P=0.0151$ (H).

tively). First, we noted that cluster I sites, which represent the majority of all sites, had comparable accessibility at baseline, but a great increase in accessibility after IFN treatment. The majority of IFN- $\gamma$-responsive chromosomal regions became more accessible in only ARID1A-proficient cells, not ARID1A-deficient cells (Figure 3, B and C). We also observed a small set of chromosomal regions (cluster III) with increased accessibilities after IFN- $\gamma$ exposure in ARID1A-deficient cells as compared with proficient cells. Those sites tended to have closed chromatin at baseline. We further performed motif and target gene prediction analysis, and validated that IFN- $\gamma$-responsive sites were highly enriched with IRF2 motifs and associated with IFN-regulated genes (Figure 3D). In addition, we identified that the IRF 2 motif was also enriched in the open chromosomal regions affected by ARID1A deficiency (Supplemental Table 5). These global trends were recapitulated at the level of key individual loci. Specifically, we found significant reduction of chromosomal accessibility at the Th1-type chemokine locus in ARID1A-deficient cells upon IFN stimulation. Although ARID1A can be enriched at the enhancer sites in HCT116 cell lines and mouse models $(35,36)$, we observed strong peaks on the transcription start sites (TSS) of CXCL9, CXCL10, and CXCL11 genes, all of which were decreased upon ARID1A loss (Figure 3E). Similar results were observed in other Th1-type chemokine genes, CCL2 and CCL5 (Supplemental Figure 3A). To further underscore the functional consequences of these chromatin openings, we investigated the overlap between ATAC-Seq and RNA-Seq data sets. Of 
A

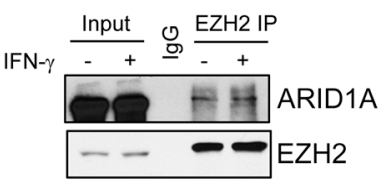

B

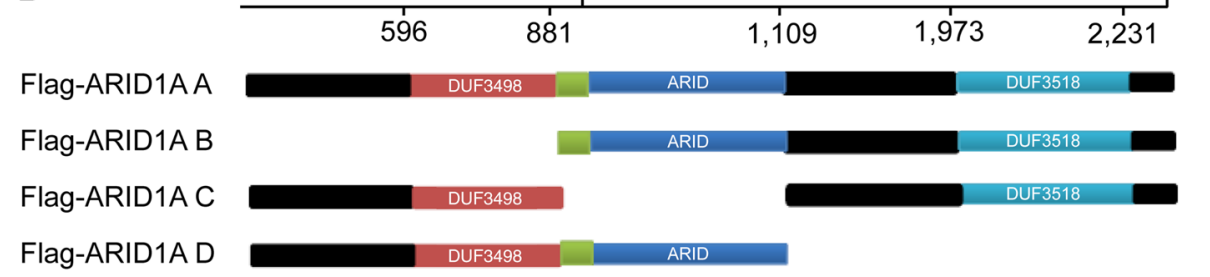

C

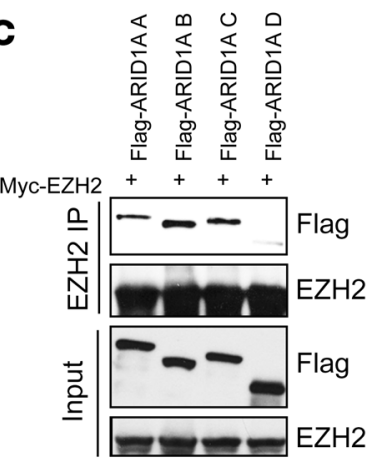

D

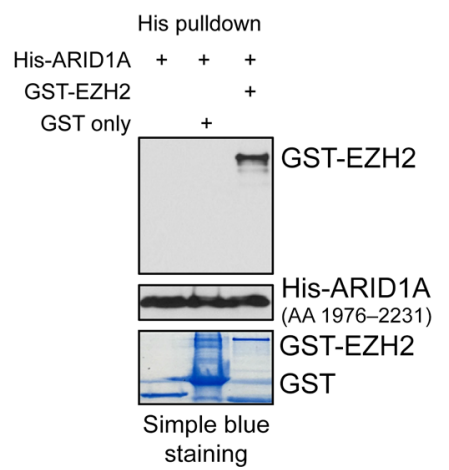

E

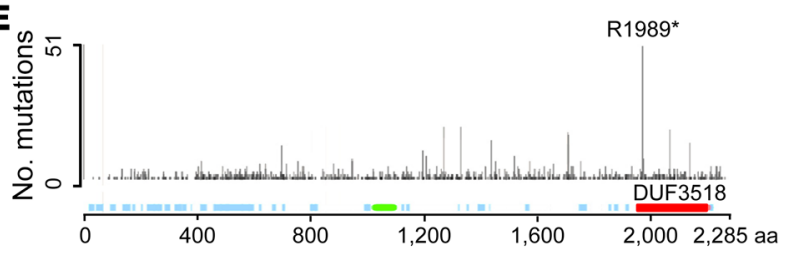

$\mathbf{F}$

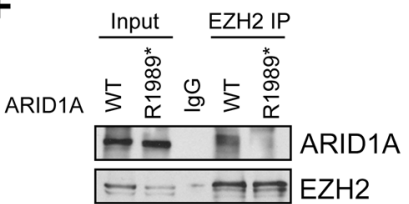

Figure 4. ARID1A biochemically interacts with EZH2. (A) Interaction between ARID1A and EZH2 in primary high-grade serous ovarian cancer cells (OC8). Endogenous EZH2 was immunoprecipitated with anti-EZH2 and ARID1A was probed with Western blot. One of 3 is shown. (B) Schematic representation of the full-length ARID1A (ARID1A A) and multiple ARID1A mutants (ARID1A B, C, D). The full-length ARID1A and mutants were used for co-interaction analyses. (C) Interaction between ARID1A and EZH2. Myc-EZH2 and Flag-ARID1A full-length and mutants were ectopically expressed in HEK293T cells, followed by EZH2 immunoprecipitations and immunoblotting with Flag and EZH2 antibodies. Inputs are shown in bottom panels. One of 3 is shown. (D) In vitro binding of ARID1A to EZH2. Recombinant His-ARID1A C-terminal DUF 3518 domain partial protein (AA1976-2231) was incubated with GST-EZH2 recombinant protein, followed by His tag pulldown and immunoblotting with GST antibody. Inputs are shown in bottom panels. One of 3 is shown. (E) R1989* hotspot mutation of ARID1A in all types of cancer. Image was adopted from Cosmic website (https://cancer.sanger.ac.uk/cosmic), and double-checked in Cbioportal. (F) Interaction between ARID1A R1989* mutant and EZH2. HEK293T cells were transfected with WT Flag-ARID1A and R1989* mutant expressing plasmids, followed by EZH2 immunoprecipitation and immunoblotting with ARID1A and EZH2 antibodies. One of 3 is shown.

all IFN- $\gamma$-responsive genes that were regulated by ARID1A, identified from RNA-Seq, 51\% harbored chromosomal sites that had differential accessibilities after ARID1A loss within $5 \mathrm{~kb}$ of their TSS. These data suggest that ARID1A regulates IFN- $\gamma$-responsive genes at least partially via controlling the chromatin accessibilities of their promoters (Figure 3F and Supplemental Table 6). To validate this observation in different human cancers, we additionally studied a primary human high-grade serous ovarian cancer cell line (OC8) and an established human melanoma cell line (CHL1) and their ARID1A-deficient cells (OC8-KO and CHL1-KO). We stimulated these cells with IFN- $\gamma$ and conducted ATAC-Seq analysis in OC8 and OC8-KO (Supplemental Figure 3B) and CHL1 and CHL1-KO (Supplemental Figure 3C). Again, ARID1A deficiency resulted in reduced chromatin accessibilities to IFN- $\gamma$-upregulated signaling genes in both OC8-KO and CHL1-KO as compared with parental cells (Supplemental Figure 3, B and C).

In addition to the established tumor cell lines, we also examined ATAC-Seq data in primary human melanoma and colon cancer tissues (37). We observed significant correlations between ARID1A gene expression and the averaged ATAC-Seq peak intensities for Th1-type chemokines (CXCL9 and CXCL10) in patients with melanoma (Figure 3, $\mathrm{G}$ and $\mathrm{H}$ ) and patients with colon cancer (Supplemental Figure 3, D and E, and ref. 37). This correlation remained significant after excluding colon cancer patients with MMR deficiency (Supplemental Figure 3, F and
G). These data provide strong evidence that ARID1A regulates expression of IFN- $\gamma$-signaling genes at least partially through controlling chromatin accessibility.

In order to verify that the ARID1A-associated BAF complex was recruited to the Th1-chemokine promoter sites, we carried out the chromatin immunoprecipitation (ChIP) assay and examined ARID1A and BAF component BAF155 (SMARCC1) recruitment on the promoters of CXCL9 and CXCL10 in cancer cells in response to IFN- $\gamma$. We found that IFN- $\gamma$ stimulation resulted in high occupancies of ARID1A (Supplemental Figure 3, H-M) and SMARCC1 (Supplemental Figure 3, N and O) on the promoters of CXCL9 (Supplemental Figure 3, H-J and N) and CXCL10 (Supplemental Figure 3, K-M and O) in ARID1A-proficient DLD-1 colon cancer cells, OC8 ovarian cancer cells, and A375 melanoma cancer cells (Supplemental Figure 3, H-M), whereas SMARCC1 occupation on CXCL9 and CXCL10 promoter was largely reduced in ARID1A-knockout OC8 cells compared with WT ARID1A OC8 cells in response to IFN- $\gamma$ stimulation (Supplemental Figure 3, N and O). Overall, we conclude that ARIDIA is largely required for IFN- $\gamma$-induced gene expression and loss of ARID1A compromises chromatin accessibility at the IFN-responsive gene loci.

ARID1A biochemically, genomically, and functionally interacts with EZH2. Next, we studied how ARID1A regulates IFN gene expression. Early genetic studies in Drosophila suggested a potential interaction between the SWI/SNF complex and PRC2 $(22,23)$. 
A
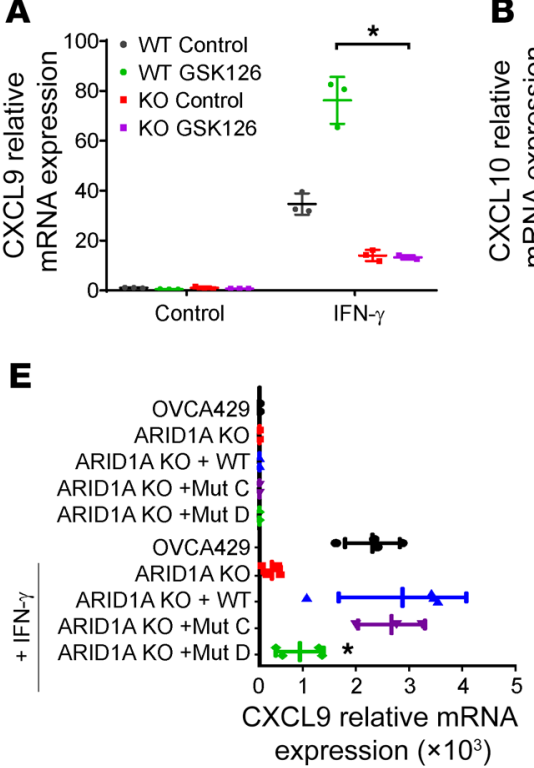

G

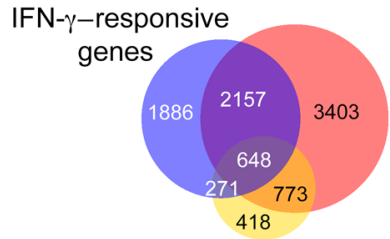

GSK126 affected genes

in IFN- $\gamma$-stimulated condition condition condition

$$
\text { tion }
$$
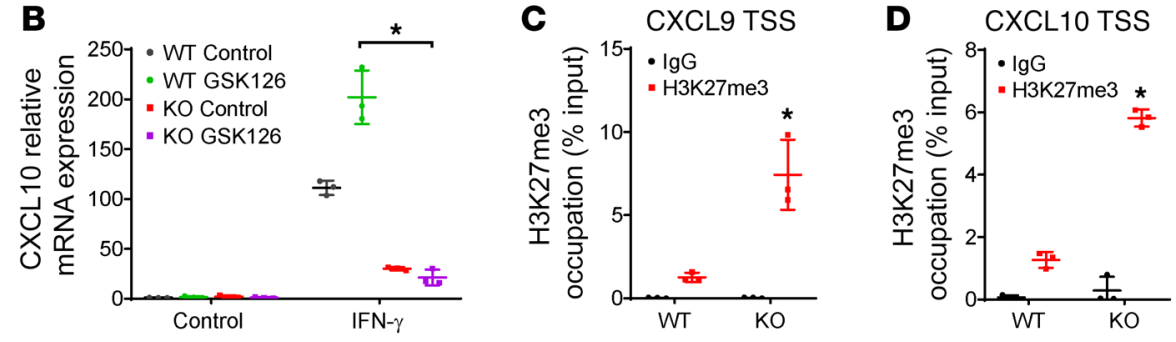

$\mathbf{F}$

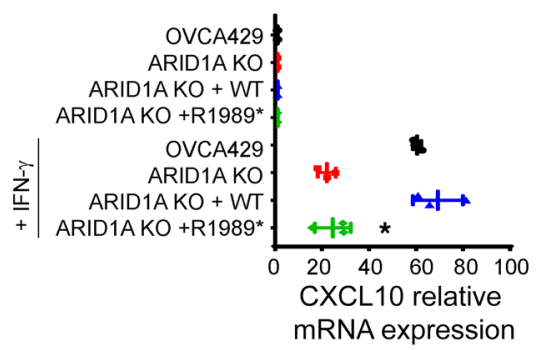

ARID1A knockout affected genes in IFN- $\gamma$-stimulated

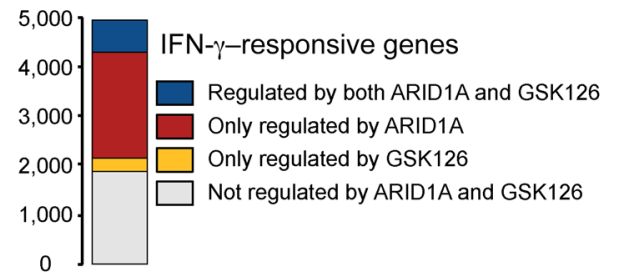

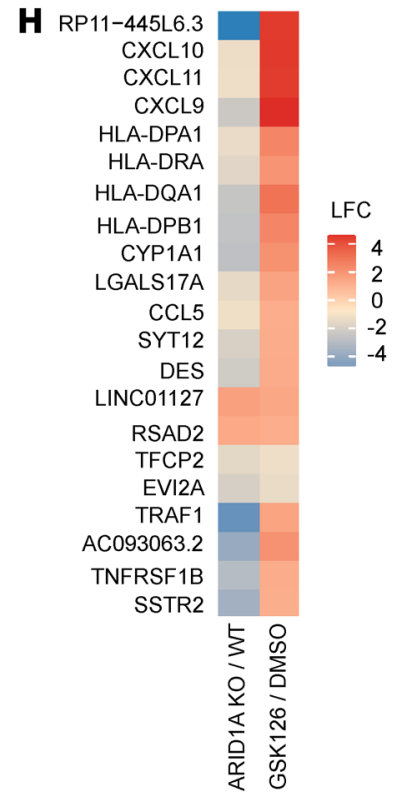

Figure 5. ARID1A functionally interacts with EZH2. (A and B) Effect of ARID1A on EZH2-mediated Th1-type chemokine repression in ovarian cancer cells. ARID1A WT or knockout OC8 cells were pretreated with GSK126, following IFN- $\gamma$ treatment for 8 hours. CXCL9 (A) and CXCL10 (B) expression were quantified by real-time PCR. Mean $\pm \mathrm{SD}, n=3$ with repeats, ${ }^{*} P=0.0032$ (A), ${ }^{*} P=0.0014$ (B), Student's 2-tailed $t$ tests. (C and D) Effect of ARID1A on H3K27me3-mediated Th1-type chemokine repression in ovarian cancer cells. ARID1A WT or knockout OC8 cells were treated with IFN- $\gamma$ for 6 hours. H3K27me3 ChIP was performed. H3K27me3 levels on the promoters of CXCL9 and CXCL10 were normalized to the input. Mean \pm SD, $n=3-4,{ }^{*} P$ $=0.00155$ (C), ${ }^{*} P=0.00003$ (D), Student's 2-tailed $t$ tests. (E) Effect of ARID1A C-terminal truncation on CXCL9 gene expression in ovarian cancer cells. ARID1A-knockout OVCA429 cells were transfected with full-length ARID1A, ARID1A mutant C, and ARID1A mutant D (ARID1A C-terminal truncation) (see Figure 4B). CXCL9 expression was quantified by real-time PCR. ( $n=3,{ }^{*} P=0.0017$, Student's 2-tailed $t$ tests). (F) Effect of ARID1A R1989* mutation on CXCL10 gene expression in ovarian cancer cells. ARID1A-knockout OVCA429 cells were transfected with WT ARID1A or ARID1A R1989* mutants and stimulated with IFN- $\gamma$ for 12 hours. CXCL10 expression was quantified by real-time PCR. ( $n=3,{ }^{*} P=0.028$, Student's $t$ tests). (C) Venn diagram depicting overlap between genes significantly regulated following IFN- $\gamma$ stimulation (blue), ARID1A knockout (red), or GSK126 treatment (yellow) in OVCA-429 cells. Stacked bar plot depicting the distribution of ARID1A- or GSK126- regulation status of IFN- $\gamma$-responsive genes. (H) Log $_{2}$ fold change (LFC) of top IFN- $\gamma$-responsive genes that are significantly regulated following ARID1A knockout or GSK126 treatment, $n=2$.

EZH2, a key PRC2 component, represses Th1-type chemokine expression in human ovarian carcinoma and colon carcinoma $(27,28)$. In addition, transcriptome analysis indicated that the top pathway associated with ARID1A was Th1-type immune response (Figure 1A). The data raised a question of whether ARID1A physically interacts with EZH2 to functionally antagonize the repressive role of EZH2 in IFN-responsive genes in human cancer cells. We used an anti-EZH2 mAb to immunoprecipitate endogenous $\mathrm{EZH} 2$ in OC8 cells. We detected an interaction between endogenous EZH2 and ARID1A in OC8 (Figure 4A). This interaction was IFN- $\gamma$ independent (Figure 4A). We confirmed the interaction between endogenous ARID1A and EZH2 in human HCT116 colon cancer cells (Supplemental Figure 4A). To further investigate this interaction in vivo, we inoculated OVCA429 cells into $\mathrm{NOD} /$ scid IL2R $\gamma^{\text {null }}$ (NSG) mice and established ovarian cancer.
Then, we isolated the established ovarian tumor and confirmed an interaction between ARID1A and EZH2 in vivo (Supplemental Figure 4B). Next, we thought to determine which domain of ARID1A interacted with EZH2. To address this, we generated a series of ARID1A mutants, transfected HEK293T cells with plasmids encoding WT ARID1A or ARID1A mutants (Figure 4B) - along with plasmids encoding WT EZH2 (Figure 4C) - and performed immunoprecipitation experiments. We detected an interaction between WT EZH2 and WT ARID1A in HEK293T cells (Figure 4C). Furthermore, ARID1A interacted with EZH2 via its C-terminal region, which contained amino acids from 1109 to 2285 (Figure 4, B and C). Using the recombinant DUF3518 domain of ARID1A covering amino acids 1976 to 2231 and recombinant GST-EZH2 protein, we confirmed that the DUF3518 domain of ARID1A interacts with EZH2 (Figure 4D). A search of the TCGA 
A
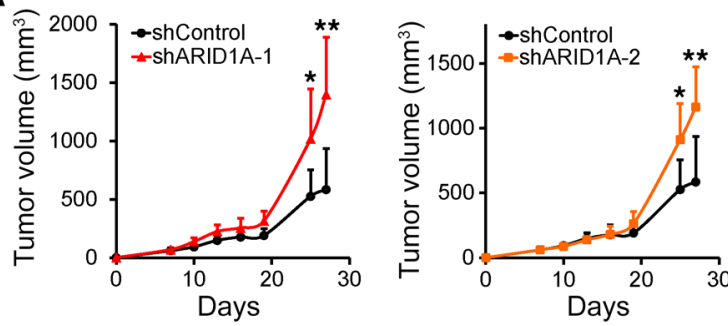

D

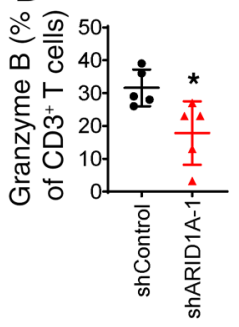

E

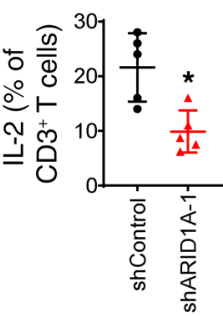

$\mathbf{F}$
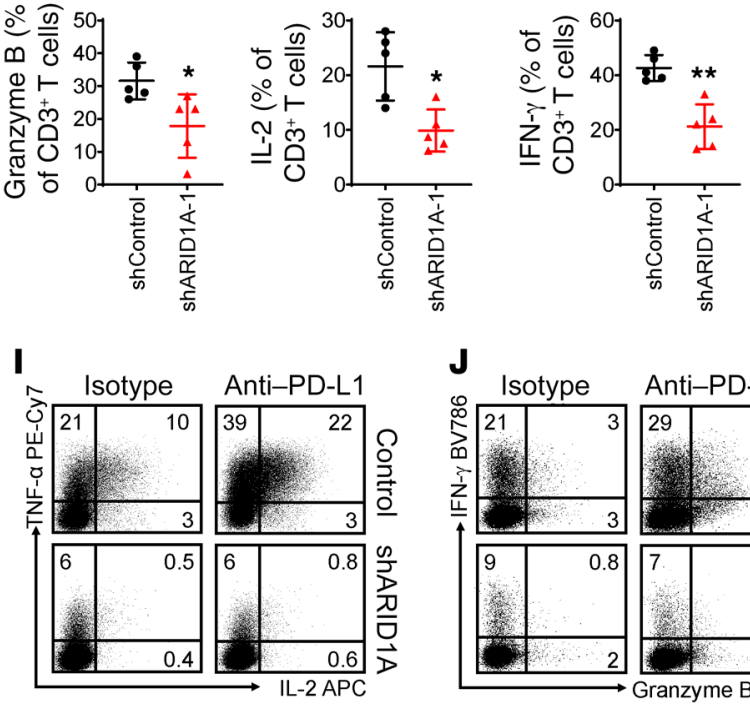

B

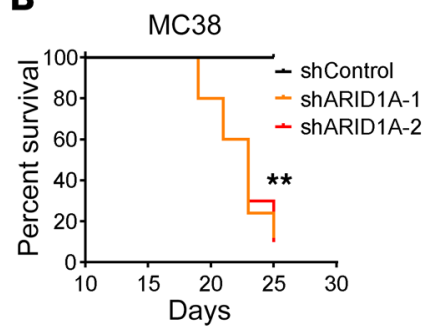

G

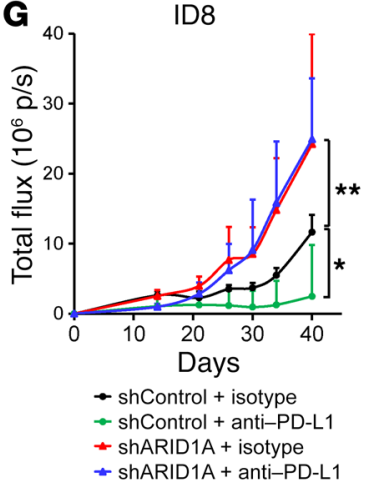

C
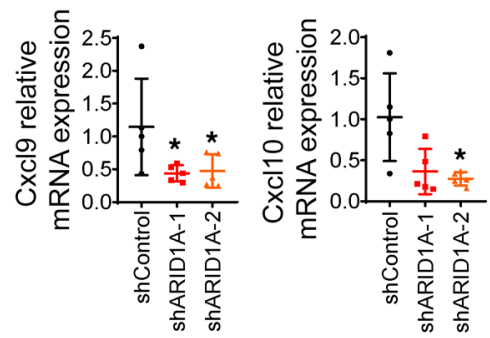

H

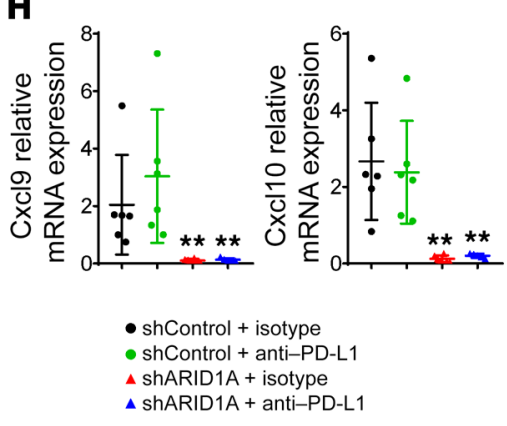

Figure 6. ARID1A regulates spontaneous tumor immunity in vivo. (A and B) Effects of ARID1A on MC38 tumor growth and mouse survival in C57BL/6 mice. Mice were inoculated with MC38 expressing shARID1As and control vectors (the same control vectors for shARID1A-1 and shARID1A-2). Tumor volume $(\mathbf{A})$ and mouse survival (B) were monitored. Mean \pm SD, $n=7-8$, Mann-Whitney $U$ test $(\mathbf{A}) .{ }^{*} P<0.05 ;{ }^{* *} P<0.01$. Kaplan-Meier analysis (B). (C) Effect of ARID1A on MC38 tumor chemokine expression. CXCL9 and CXCL10 transcripts were quantified by real-time PCR in shARID1A- and vector-expressing MC38 tumors in vivo. Mean \pm SD, $n=5$, Mann-Whitney $U$ test; $C X C L 9:{ }^{*} P=0.0317$; CXCL10: ${ }^{*} P=0.0159$. (D-F) Effect of ARID1A on MC38 tumor-infiltrating T cell function. Tumor-infiltrating granzyme $\mathrm{B}^{+}(\mathbf{D}), \mathrm{IL}-2^{+}(\mathbf{E})$, and IFN- $\gamma^{+}(\mathbf{F}) \mathrm{T}$ cells were analyzed on day 17 . Gated on $\mathrm{CD} 45^{+} \mathrm{CD} 3^{+} \mathrm{T}$ cells. Mean $\pm \mathrm{SD}, n=5$, Mann-Whitney $U$ test, ${ }^{*} P<0.05$, ${ }^{* *} P<0.01$. (C) Effect of ARID1A on ID8 ovarian cancer growth in C57BL/6 mice. Mice were inoculated with luciferase-ID8 expressing shARID1A and control vectors. Tumor volume was monitored. Mean $\pm \mathrm{SD}, n=5-6$, Mann-Whitney $U$ test, ${ }^{*} P<0.05$; ${ }^{* *} P<0.01$. (H) Effect of ARID1A on ID8 tumor chemokine expression. CXCL9 and CXCL10 transcripts were quantified by real-time PCR in shARID1A- and vector-expressing ID8 tumors in vivo. Mean $\pm S D, n=5-6$, Mann-Whitney $U$ test, ${ }^{* *} P<0.01$. (I and J) Effect of ARID1A in ID8 tumor-infiltrating T cell function. Tumor-infiltrating TNF- $\alpha^{+}$and IL- $2^{+} \mathrm{CD4}^{+}(\mathrm{I})$ and IFN- $\gamma^{+}$and granzyme $\mathrm{B}^{+} \mathrm{CD} 8^{+}(\mathrm{J})$ T cells were analyzed. $n=5-6$.

database for ARID1A mutation status in cancers revealed that an R1989* nonsense mutation in the DUF3518 domain is a hotspot mutation in a variety of cancer types (Figure $4 \mathrm{E}$; https://cancer. sanger.ac.uk/cosmic/gene/analysis?ln=ARID1A) (38). Moreover, we made an ARID1A mutant with the R1989* mutation and detected a compromised interaction between EZH2 and the R1989* mutant (Figure 4F). The data, along with our subsequent functional studies, suggest a clinical relevance of this hotspot mutation in human tumor immunity.

After examining their biochemical interaction, we evaluated the functional significance of the ARID1A-EZH2 interaction in the regulation of IFN- $\gamma$-responsive genes in human tumor cells. We hypothesized that ARID1A functionally antagonizes the repressive role of EZH2 in IFN-responsive genes in human cancer cells. GSK126 specifically inhibits EZH2 methyltransferase activity (39). We stimulated ARID1A WT and knockout OC8 cells with IFN- $\gamma$ in the presence of GSK126. In line with our previous report (28), GSK126 treatment promoted CXCL9 and CXCL10 expression in
ARID1A WT cells. However, this effect was largely diminished in ARID1A-knockout ovarian cancer cells (Figure 5, A and B). Similar results were obtained in ARID1A WT (OVCA249) and deficient (AC17, AC25) OCCC cells (Supplemental Figure 5, A and B).

Furthermore, we performed ATAC-Seq in OVCA429 and OVCA429 ARID1A-knockout cells upon IFN- $\gamma$ stimulation in the presence of GSK126. We observed that the chromatin accessibility of IFN- $\gamma$-responsive genes was moderately lower in ARID1A-knockout cells compared with ARID1A WT cells (Supplemental Figure 5C). This result indicates that ARID1A deficiency led to poorer chromatin accessibility even in the presence of GSK126. To additionally explore the antagonistic effect between EZH2 and ARID1A, we carried out ChIP analysis and examined H3K27 trimethylation status on the promoters of CXCL9 and CXCL10. ARID1A knockout or mutant showed higher levels of H3K27me3 occupancy at the promoters of CXCL9 and CXCL10 than ARID1A-proficient ovarian cancer cells after IFN- $\gamma$ treatment (Figure 5, C and D, and Supplemental Figure 5, D and E). EZH2 


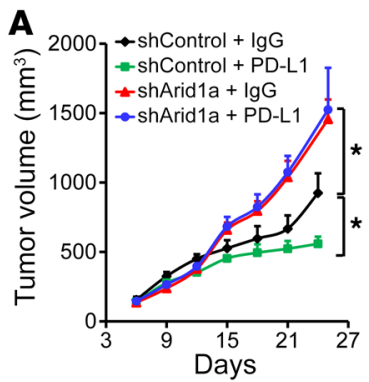

D

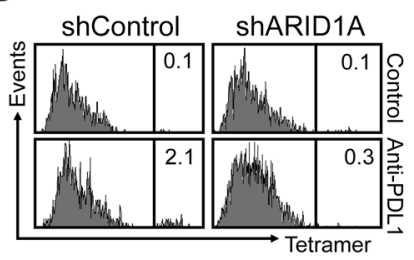

G

Riaz N/Timothy Chan cohort - 52 patients

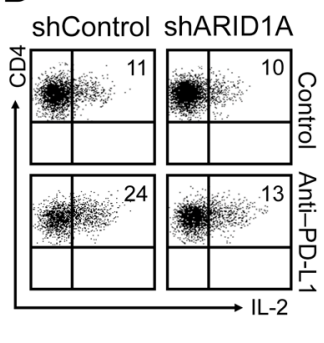

E

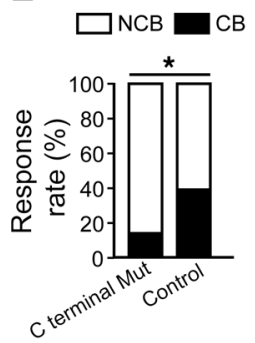

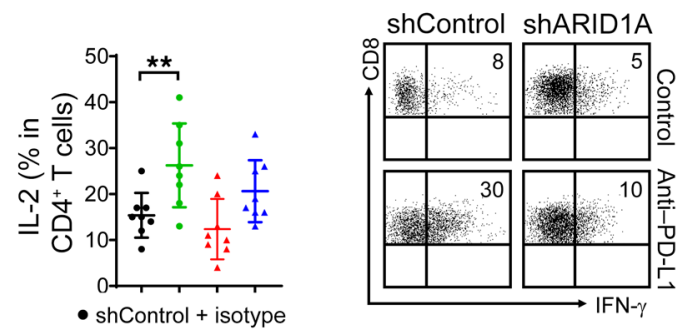

- shControl + isotype

- shControl + anti-PD-L1

$\triangle$ shARID1A + isotype

\ shARID1A + anti-PD-L1

F

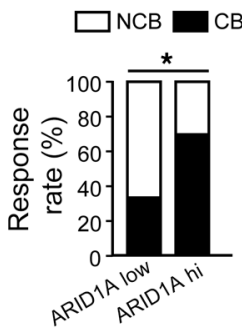

C

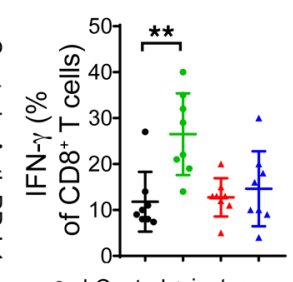

- shControl + isotype

- shControl + anti-PD-L1

$\triangle$ shARID1A + isotype

- shARID1A + anti-PD-L1

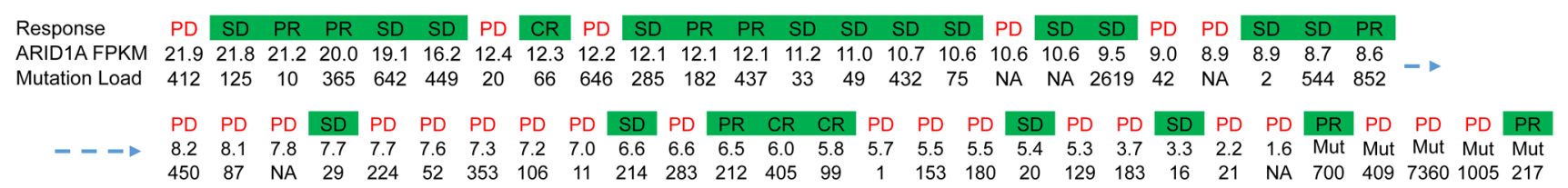

ARID1A gene expression Levels

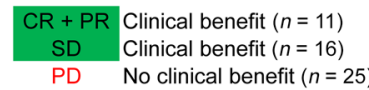

I Enriched biological functions in $\mathrm{CB}$ patients

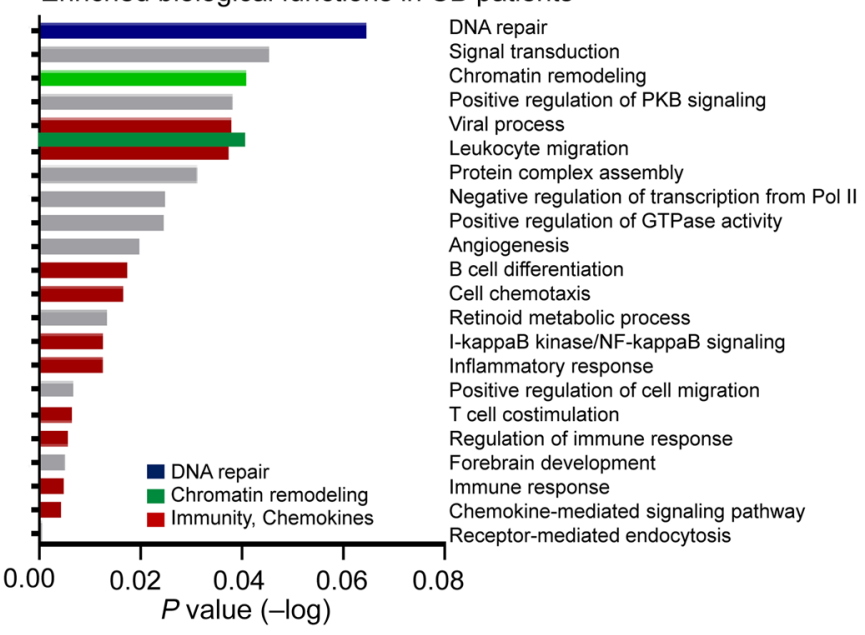

Figure 7. ARID1A gene status affects checkpoint therapy. (A-D) Effect of ARID1A on anti-PD-L1 therapy in MC38-bearing mice. Mice bearing shARID1A and vector MC38 tumors were treated with anti-PD-L1 or isotype. (A) Tumor volume was monitored. (B-D) Tumor IL-2+CD4+ (B), IFN- $\gamma^{+}$CD8 ${ }^{+}$(C), and Adpgk-specific CD8 ${ }^{+}$(D) T cells were analyzed on day 25. One of 6 experiments. Mean \pm SD, Mann-Whitney $U$ test. (E) Effect of ARID1A mutations on immunotherapeutic efficacy in metastatic melanoma patients with $(n=14)$ or without $(n=268)$ ARID1A C-terminal mutations. Response rate is shown in patients with clinical benefits $(C B, n=109)$, including complete response $(C R)$, partial response $(P R)$, and stable disease (SD), and progressive patients (PD) (nonclinical benefits, NCB, $n=173$ ). One-sided $\chi^{2}$ test, $P=0.0326$. (F and $\mathbf{G}$ ) Effect of ARID1A levels on immunotherapeutic efficacy in 52 metastatic melanoma patients with $47 \mathrm{WT}$ and 5 mutated ARID1A. (F) Response rate is shown in patients with low $(n=26)$ and high $(n=26)$ ARID1A expression. ARID1A-mutated patients were placed in the ARID1A low group. Two-sided $\chi^{2}$ test, $P=0.0125$. (C) Response status with corresponding specific ARID1A fragments per kilobase of transcript per million mapped reads is shown. (H) Effect of tumor mutation load (TMB) on immunotherapeutic efficacy. WT ARID1A melanoma patients were divided into high-TMB $(n=29)$ and low-TMB $(n=22)$ groups. Response rate was analyzed in patients with high and low TMB. In high-TMB group, 14 and 15 patients expressed, respectively, low and high ARID1A. In low-TMB group, 11 and 11 patients expressed, respectively, low and high ARID1A. Cutoff value: 100 mutations (31). Two-sided $\chi^{2}$ test, $P<0.0001$. (I) Effect of anti-PD-1 on biological pathways in melanoma patients. Differential gene expression between CB and NCB groups was entered for DAVID pathway analysis $(65) .{ }^{*} P<0.05 ;{ }^{* *} P<0.01$. 

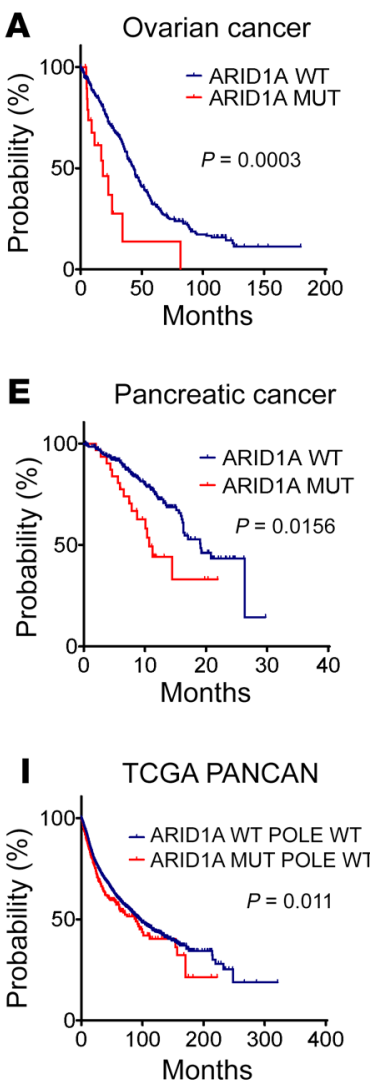
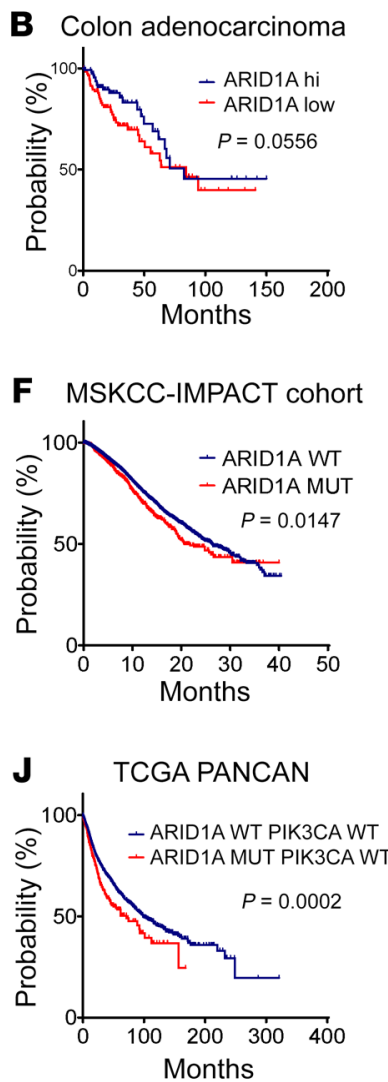
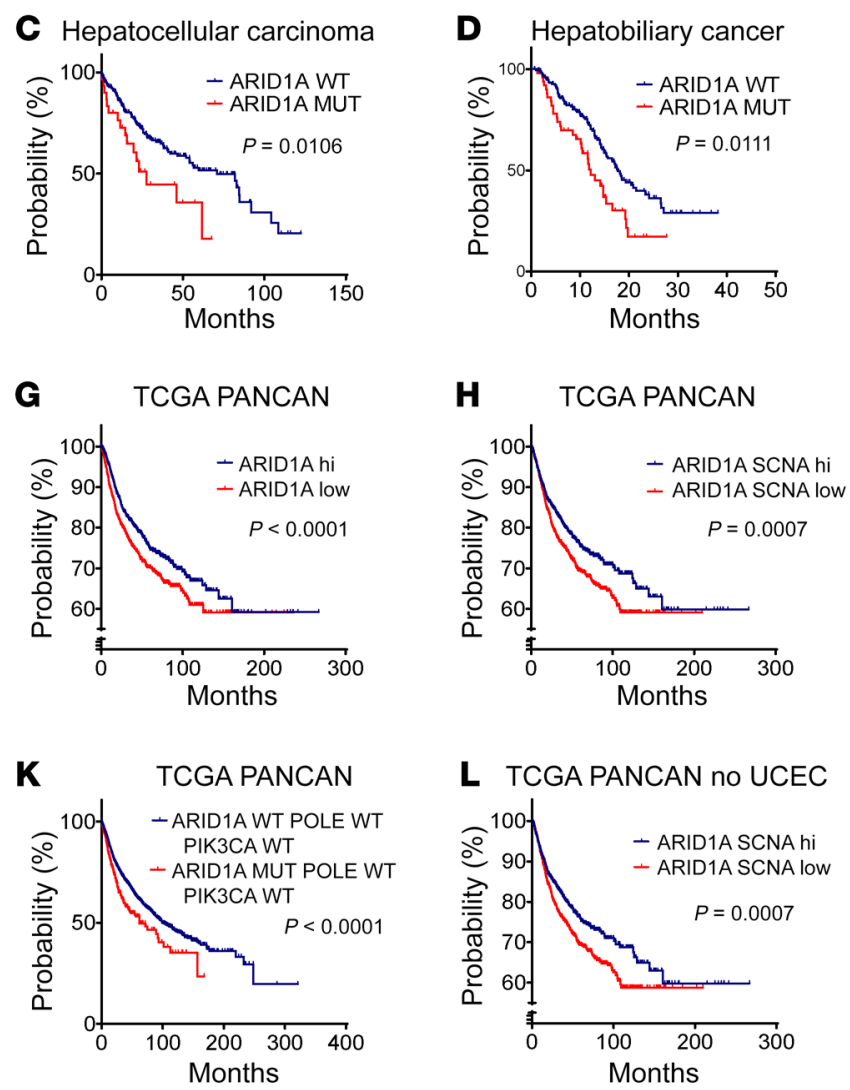

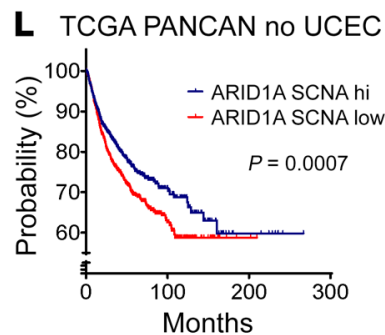

Figure 8. ARID1A gene status affects clinical outcome. (A) Overall survival (OS) of WT $(n=401)$ and mutated $(n=20)$ ARID1A ovarian cancer patients in TCCA and MSKCC-IMPACT. Log-rank test, $P=0.0003$. (B) OS of WT patients, ARID1A mRNA high $(n=119)$ and low $(n=119)$ colon adenocarcinoma patients in TCGA. $P=0.0556$. (C) OS of WT $(n=341)$ and mutated $(n=31)$ ARID1A hepatocellular carcinoma patients. $P=0.0106$. (D) 0S of WT ( $n=190)$ and mutated $(n=49)$ ARID1A hepatobiliary cancer patients. $P=0.0111$. (E) OS of WT $(n=328)$ and mutated $(n=38)$ ARID1A pancreatic cancer patients. $P=0.0156$. (F) OS of WT ( $n=6913)$ and mutated $(n=661)$ ARID1A patients in MSKCC-IMPACT. $P=0.0147$. (G) Disease-free survival (DFS) of high $(n=2098)$ and low $(n=$ 2098) tumor ARID1A transcripts in MSKCC-IMPACT. $P<0.0001$. (H) DFS of high $(n=2034)$ and low $(n=2034)$ tumor WT ARID1A somatic copy numbers in patients in TCGA PANCAN. $P=0.0007$. (I) OS of WT $(n=7979)$ and mutated $(n=644)$ ARID1A patients in TCGA PANCAN. Patients with POLE mutations were excluded. $P=0.011$. (J) OS of WT $(n=6897)$ and mutated $(n=457)$ ARID1A patients in TCGA PANCAN. Patients with PIK3CA mutations were excluded. $P=0.0002$. (K) OS of WT $(n=6745)$ and mutated $(n=401)$ ARID1A patients in TCGA PANCAN. Patients with PIK3CA or POLE mutations were excluded. $P<0.0001$. (L) DFS of high $(n=1957)$ and low $(n=1958)$ WT ARID1A somatic copy numbers in TCGA PANCAN patients. UCEC were excluded. $P=0.0007$.

mediates CXCL9 and CXCL10 repression in tumor cells through H3K27me3 (28). The data suggest that ARID1A functionally antagonizes EZH2-mediated IFN- $\gamma$ gene repression.

Given that the DUF3518 domain of ARID1A is essential for its interaction with EZH2, we explored whether the DUF3518 domain is functionally required for ARID1A to antagonize EZH2-mediated IFN- $\gamma$ signaling gene repression. To this end, we generated an ARID1A mutant with DUF3518 domain deletion (Figure 4, B and C) to test whether WT ARID1A or different ARID1A mutants could rescue defective CXCL10 gene expression in ARID1A-knockout ovarian cancer cells. We found that WT ARID1A and ARID1A mutant C, but not ARID1A mutant D (C-terminal mutant) (Figure 4B), rescued CXCL10 gene expression (Figure 5E). The data indicate that the DUF3518 domain is critical for ARID1A to functionally antagonize EZH2-mediated IFN- $\gamma$ gene repression. To assess the functional importance of the R1989* nonsense mutation, the clinical hotspot mutation of ARID1A, we made the R1989* mutant and tested its role (Figure 4F) in tumor cell IFN- $\gamma$ response. The R1989* mutant failed to rescue CXCL10 deficiency in ARID1A-knockout ovarian cancer cells in response to IFN- $\gamma$ (Figure 5F).
Both ARID1A deficiency and EZH2 repression (GSK126) altered IFN- $\gamma$-responsive gene expression (Figure 5G). We explored the potential coordinative effect of ARID1A and EZH2

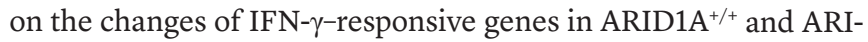

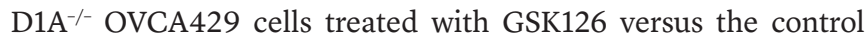
(DMSO). Overall, more than two-thirds of GSK126-affected genes were overlapped with ARID1A-affected genes (Figure 5G and Supplemental Table 7). Interestingly, the majority of these gene changes were in opposite directions (Supplemental Figure 5, F and G). Th1-type chemokines, including CCL5, CXCL9, CXCL10, and CXCL11, were among the top genes differentially regulated by both EZH2 and ARID1A (Figure 5H and Supplemental Table 7). Thus, ARID1A biochemically interacts with EZH2 and functionally antagonizes EZH2-mediated IFN- $\gamma$ gene repression in tumor cells via its carboxyl domain. Altogether, these data suggest a critical interplay between ARID1A and EZH2 to regulate a set of IFN-responsive genes.

ARID1A regulates spontaneous tumor immunity in vivo. Given the role of ARID1A in the regulation of IFN signaling in tumors, particularly Th1-type chemokines, we hypothesized that ARID1A 
affects antitumor immunity in vivo by controlling effector $\mathrm{T}$ cell tumor trafficking via CXCL9 and CXCL10. To test this hypothesis, in the first experimental setting, we treated MC38 colon cancerbearing mice with neutralizing anti-CXCR3 mAb. CXCR3 is the receptor for CXCL9 and CXCL10. Anti-CXCR3 mAb treatment resulted in reduced $\mathrm{CD}^{+} \mathrm{T}$ cells in the tumor microenvironment (Supplemental Figure 6A) and increased tumor growth (Supplemental Figure 6B) as compared with the control. Thus, we validated a critical role of CXCL9 and CXCL10 in T cell tumor trafficking in MC38 tumor-bearing mice.

In the second experimental setting, we established 2 ARID1A knockdown MC38 cell pools with specific shRNAs against ARID1A (shARID1A-1 and shARID1A-2) (Supplemental Figure 6C). shRNAmediated knockdown of ARID1A did not affect tumor growth in vitro (Supplemental Figure 6D) or in vivo in NSG mice (Supplemental Figure 6E), but caused increased tumor volume (Figure 6A) and shortened mouse survival (Figure 6B) in immune-competent C57BL/6 mice. Thus, knockdown ARID1A negatively affects spontaneous antitumor immunity.

In the third experimental setting, we depleted $\mathrm{CD} 8^{+} \mathrm{T}$ cells with anti-mouse CD8 $\mathrm{mAb}$ in mice bearing 2 different shARID1A MC38 cell pools. Depletion of $\mathrm{CD}^{+} \mathrm{T}$ cells erased the difference in tumor growth between mice bearing WT ARID1A and 2 different shARID1A tumor cell pools (Supplemental Figure 6, F and $G$ ). Furthermore, the levels of Th1-type chemokines CXCL9 and CXCL10 (Figure 6C) and T cell effector molecules, including granzyme B, IL-2, and IFN- $\gamma$ (Figure 6, D-F), were decreased in the tumor microenvironment in mice bearing ARID1A knockdown MC38 compared with controls.

To validate these results in a different model system, we knocked down ARID1A in ID8 mouse ovarian cancer cells with specific shARID1A (Supplemental Figure 6H). IFN- $\gamma$ stimulation did not affect ARID1A knockdown efficiency (Supplemental Figure $6 \mathrm{H})$. Again, shARID1A ID8 tumors grew faster than WT ID8 tumors in C57 BL/6 mice (Figure 6G and Supplemental Figure 6I). The levels of Th1-type chemokines (Figure 6H), effector molecules expressed in $\mathrm{CD}^{+} \mathrm{T}$ cells (Figure 6, I and J), in $\mathrm{CD}^{+}$(Supplemental Figure 6J), and $\mathrm{CD}^{+}$(Supplemental Figure 6K) $\mathrm{T}$ cell subsets were decreased in the shARID1A ID8 tumor microenvironment as compared with the WT ID8 tumor. Thus, ARID1A deficiency causes poor tumor Th1-type chemokine expression and $\mathrm{T}$ cell tumor infiltration, and impedes spontaneous tumor immunity in vivo.

ARID1A gene status affects checkpoint therapy-induced tumor immunity. We explored whether ARID1A affected checkpoint blockade-induced immunity and therapeutic response in animal models and in patients with cancer. First, we treated mice bearing WT ARID1A and shARID1A MC38 (Supplemental Figure 5, A and B) and ID8 (Figure 6G) cancer cells with anti-PD-L1 mAb. Treatment with PD-L1 mAb slowed down tumor growth in mice bearing control vector-expressing MC38 (Supplemental Figure 6B) and ID8 (Figure 6G) cancer cells. However, knockdown of tumor ARID1A abolished the antitumor effect of PD-L1 mAb in mice bearing shARID1A MC38 (Figure 7A) and ID8 (Figure 6G) tumors. In line with this, anti-PD-L1 mAb treatment enhanced the expression levels of CXCL9 and CXCL10 (Figure 6H) of IL-2, TNF- $\alpha$, IFN- $\gamma$, and granzyme B in T cells (Figure 6, I and J) and in $\mathrm{CD}^{+}$(Supplemental Figure 6J) and $\mathrm{CD}^{+} \mathrm{T}$ cell subsets (Supplemental Fig- ure 6K) in the WT ID8 tumor microenvironment, but not in the shARID1A-ID8 tumor microenvironment.

As confirmatory experiments, we observed that in MC38 tumor-bearing mice, tumor-infiltrating $\mathrm{IL}-2^{+} \mathrm{CD} 4^{+} \mathrm{T}$ cells (Figure $7 \mathrm{~B}$ ) and IFN- $\gamma^{+} \mathrm{CD} 8^{+} \mathrm{T}$ cells (Figure $7 \mathrm{C}$ ) were higher in mice bearing control vector-expressing MC38, compared with mice bearing shARID1A-expressing MC38 tumors. Again, anti-PD-L1 therapy increased tumor-infiltrating IL $-2^{+} \mathrm{CD} 4^{+} \mathrm{T}$ cells (Figure 7B) and IFN $-\gamma^{+} \mathrm{CD}^{+} \mathrm{T}$ cells (Figure 7C) in mice with control vector-expressing MC38, but not in mice with shARID1A-expressing MC38 tumors. Furthermore, anti-CXCR3 mAb treatment abolished the antitumor effect of anti-PD-L1 mAb in mice bearing WT MC38, as shown by decreased tumor-infiltrating $\mathrm{CD}^{+} \mathrm{T}$ cells (Supplemental Figure 6A) and increased tumor volume (Supplemental Figure 6B) in mice that received anti-CXCR3 and antiPD-L1 compared with mice that received anti-PD-L1 alone (Supplemental Figure 6, A and B).

MC38 cells harbor a single-epitope mutation within Adpgk protein (ASMTNRELM $\rightarrow$ ASMTNMELM), with the neoepitope presented in MHC-I H-2 $\mathrm{D}^{\mathrm{b}}$ molecules (40). Neoantigen tetramer assay showed that PD-L1 mAb treatment increased neoantigen-specific $\mathrm{CD} 8^{+} \mathrm{T}$ cells in the tumor microenvironment in mice with control shRNA tumors, but not in shARID1A tumors (Figure 7D and Supplemental Figure 7A). Neoantigen-specific CD8 ${ }^{+} \mathrm{T}$ cells were not detectable in the lymph nodes (Supplemental Figure 7B). The data suggest that ARID1A affects checkpoint blockadeinduced immunity in preclinical murine models.

Next, we explored whether ARID1A affects clinical response to checkpoint blockade in patients. Because the C-terminal portion of ARID1A interacts with EZH2 and plays a critical role in IFN- $\gamma$ response, we compared clinical responses to PD-L1/PD-1 blockade in patients with or without ARID1A C-terminal mutations. We found a nearly 3 -fold decrease in the clinical response rate of melanoma patients with ARID1A C-terminal mutations as compared with patients without C-terminal mutations (refs. 31, 41-43, and Figure 7E). We additionally evaluated the role of ARID1A gene expression levels in clinical response to checkpoint blockade in cancer patients. High levels of tumor ARID1A gene expression correlated with increased clinical response rate to anti-PD-1 therapy in patients with melanoma (ref. 31 and Figure 7, F and G). It has been suggested that ARID1A mutation is related to increased tumor mutation load and enhanced tumor immunity in a mouse model (44). To explore a potential role of tumor mutation load in ARID1A-affected human tumor immunity, we divided patients into high- versus low-mutation groups and compared their therapeutic responses to immunotherapy. We found that regardless of tumor mutation loads, the clinical response rate was lower in patients with low ARID1A expression (Figure 7H). The data suggest that tumor mutation load may not be a decisive factor for ARID1A-affected tumor immunity. In further support of this, transcriptome analysis of metastatic melanoma tissues revealed that signatures of the chromatin remodeling genes, including ARID1A, and chemokine and immune cell trafficking signaling, were enriched in patients with clinical benefits associated with immunotherapy (Figure 7I). Specific pathway analysis showed the enrichment of IFN- $\gamma$ (Supplemental Figure 7C), IFN- $\alpha$ (Supplemental Figure 7D), and T cell activation (Supplemental Figure 7E) signaling genes in patients 
with therapy-associated clinical response, whereas genes associated with mitochondria activity, catabolic process, and pyrimidine nucleotide metabolism were enriched in patients without clinical response (Supplemental Figure 7F and ref. 31). Thus, ARID1A gene status influences tumor immunity, and the impact of ARID1A on the efficacy of immune checkpoint blockade therapy may be independent of tumor mutation load.

ARID1A gene status affects patient survival. To explore the potential impact of ARID1A gene status on cancer patient outcome, we analyzed several types of human cancer in public resources in which information on both ARID1A gene status and patient outcome were available. In ovarian cancer patients, we noticed that patients with ARID1A-mutated tumors experienced a poor overall survival as compared with patients whose tumors had WT ARID1A (Figure 8A and refs. 45, 46). In colon adenocarcinoma patients with WT ARID1A, ARID1A gene expression levels revealed a positive correlation with patient overall survival (ref. 45 and Figure 8B). ARID1A mutations were also associated with poor survival in patients with hepatocellular carcinoma (Figure 8C), hepatobiliary cancer (Figure 8D), and pancreatic cancer (Figure $8 \mathrm{E}$ and ref. 46) in the MSKCC-IMPACT cohort.

Next, we analyzed a potential relationship between ARID1A genetic status and clinical outcome across diverse cancer types. In the MSKCC-IMPACT cohort with 10,945 cancer patients, we found that ARID1A mutations were significantly associated with poor overall survival (Figure $8 \mathrm{~F}$ and ref. 46). Across a TCGA pan-cancer cohort of 10,593 cancer patients (47), based on ARID1A expression, we divided patients whose tumors had WT ARID1A into high and low ARID1A expression groups. We observed that ARID1A gene expression levels were positively associated with disease-free survival (Figure 8G). In addition, patients with high ARID1A copy numbers had longer disease-free survival compared with patients with low ARID1A copy numbers (Figure $8 \mathrm{H}$ ). These data suggest that ARID1A gene status can affect patient outcome in many types of human cancer.

However, when we specifically analyzed the TCGA-Uterine Corpus Endometrial Carcinoma (TCGA-UCEC) cohort (47), in line with a previous report (44), we found that ARID1A mutations were positively associated with patient survival (Supplemental Figure 8A). Surprisingly, there was no difference in the T cell signature genes and IFN signaling pathway between ARID1A WT and mutated tumors in the TCGA-UCEC cohort (Supplemental Figure $8 \mathrm{~B})$. These results suggest that the impact of ARID1A mutations on overall survival may not be attributed to T cell immunity in UCEC patients. POLE mutations (48) and PIK3CA mutations $(16,17,49)$ frequently co-occur with ARID1A mutations $(32,48)$. We excluded patients with POLE and PIK3CA mutations and assessed the effect of ARID1A mutations on UCEC patient survival. Under this condition, ARID1A mutations had no impact on patients' overall survival (Supplemental Figure 8C). The result remained similar when microsatellite instability (MSI) patients were excluded from the analysis (Supplemental Figure 8D). Altogether, the data suggest that ARID1A genetic status has no independent impact on UCEC immune phenotype and patient outcome and hence, the effects of ARID1A are likely context dependent.

Finally, we assessed whether ARID1A genetic status remained a factor in patient survival across many types of cancer by exclud- ing the potential impact of POLE and PIK3CA mutations. To this end, we analyzed the clinical outcome of patients with WT POLE or WT PIK3CA in the TCGA pan-cancer cohort. Again, ARID1A mutation remained a negative factor for survival in patients with WT POLE (ref. 47 and Figure 8I) or WT PIK3CA (ref. 47 and Figure 8J). Concurrent exclusion of POLE and PIK3CA mutations additionally enforced a negative role of ARID1A mutations in patient survival (Figure 8K), and patients with WT ARID1A had a more than 3-year overall survival advantage compared with patients with ARID1A mutations (Figure 8K). Furthermore, patients with high ARID1A somatic copy numbers had longer disease-free survival compared with patients with low copy numbers in the pan-cancer cohort after excluding UCEC patients (Figure 8L). These data indicate that ARID1A mutations may negatively affect $\mathrm{T}$ cell immunity and are associated with poor patient survival in the majority of cancer histologies, whereas ARID1A mutations may have no independent impact on cancer immune phenotype and survival in UCEC patients.

\section{Discussion}

$\mathrm{BAF}$ and polybromo-associated $\mathrm{BAF}$ (PBAF) are 2 chromatin-remodeling complexes of the SWI/SNF family. There are many components in the BAF and PBAF complexes. The genetic, biochemical, and functional relevance of individual components in the SWI/SNF complexes is generally poorly understood in the context of cancer immunity. ARID1A is a core component in the BAF complex. BAF component mutations occur in more than $20 \%$ of human cancers, which ranks immediately after p53 (26\%) mutations $(19,50)$. ARID1A mutations are exhibited in $50 \%$ of OCCCs $(16,17)$ and $11 \%$ of colorectal adenocarcinomas $(51,52)$. Hence, in this work we have explored a potential impact of ARID1A genetic status on the key IFN signaling pathway, T cell immunity, clinical outcome, and checkpoint therapy response in multiple types of cancer, including ovarian cancer, colon cancer, and melanoma.

We have found that ARID1A mutations and reduced copy number are negatively associated with patient survival and/or checkpoint therapy responses in multiple types of cancer. Given the protective role of cancer immunity in patient outcome, we have predicted a potential causal relationship between ARID1A gene status and tumor immunity. In support of this, we have demonstrated that ARID1A is indispensable for tumor cells to express IFN signaling genes, including Th1-type chemokines, and to attract effector $\mathrm{T}$ cells toward the tumor microenvironment in cancer patients and in well-defined genetic mouse models of cancer. More importantly, ARID1A gene status correlates with IFN signaling gene signature, T cell tumor infiltration and effector function, patient survival, and clinical responses to checkpoint blockade in several types of cancer regardless of tumor mutation load. Thus, our data help to address a long-standing question of whether driver mutations can directly affect tumor $\mathrm{T}$ cell immunity and immunotherapy. Indeed, we have provided strong evidence that cancer epigenetic driver mutations such as ARID1A mutations may shape tumor immune phenotype and drive cancer immune evasion in many types of human cancer. However, additional investigations are needed to more fully understand the immunological role of each epigenetic component mutation in different types of cancer in spontaneous tumor immunity and immunotherapy-induced immunity. 
It has been proposed that high tumor mutation load may increase the probability of generating immunogenic neoantigens and be associated with enhanced immunotherapy response $(31,53)$. We have taken tumor mutation load into account to test a potential involvement of ARID1A mutations in shaping immunotherapy response. In sharp contrast to the previously proposed immune-protective role of ARID1A mutations in human tumor mutation-mediated immunity and immunotherapy response (44), we have observed that regardless of tumor mutation load, ARID1A mutations were associated with poor clinical benefit in patients who received immunotherapy. In addition to tumor mutation quantity and quality (31), the nature of immunosuppressive mechanisms, tumor epigenetics, and tumor metabolism play an important role in determining cancer immune phenotype and immunotherapy response $(28,54-59)$. We suggest that ARID1A gene status, including mutation, transcript levels, and copy number, may affect spontaneous and immune checkpoint blockadeinduced $\mathrm{T}$ cell immunity.

Unlike ARID1A, PBRM1 is a component of the PBAF complex in the SWI/SNF family (60). It has been recently reported that PBRM1 deficiency is associated with increased $\mathrm{T}$ cell tumor infiltration in the mouse B16 melanoma model (61), and its mutations may correlate with increased therapeutic response to immune checkpoint blockade in patients with renal clear cell carcinoma (62). However, it is important to determine the potential impact of other concurrent genetic alterations on these patients' survival and therapeutic outcomes. Nonetheless, this work alongside with our current report enforces the concept that chromatin remodeling complexes can participate in the regulation of cancer immune response and additionally raises the possibility that the nature of immune regulation mediated by individual components of BAF and PBAF may not be functionally identical and/or may be tissue-type dependent.

An open question had been how ARID1A regulates tumor IFN signaling gene expression. We have demonstrated that ARID1A controls IFN signaling gene chromatic accessibility. This is one layer of the mechanism by which ARID1A regulates tumor cell response to spontaneous and therapy-mediated immune stimulation. Furthermore, we have revealed that the antagonistic effect of ARID1A on EZH2 is a biochemical and functional mechanism for ARID1A-regulated IFN signaling gene expression in tumor cells. EZH2, a PRC2 component, represses Th1-type chemokine CXCL9 and CXCL10 expression and causes poor effector T cell tumor trafficking in human ovarian high-grade serous carcinoma and colon carcinoma $(27,28)$. The potential genetic interaction between the BAF complex and PRC2 has been proposed previously based on genetic studies in Drosophila $(22,23)$ and during oncogenic transformation (25). We hypothesized that ARID1A may physically interact with and functionally antagonize the role of EZH2 in human cancer cells. In line with this, we found that ARID1A interacts with EZH2 via its C-terminal and functionally antagonizes EZH2-mediated Th1-type chemokine repression, and that the DUF3518 domain of ARID1A is required for tumor cell response to IFN- $\gamma$ stimulation. In support of this, a hotspot R1989* mutation within the DUF3518 domain in ARID1A has been identified in human cancers (38). Indeed, this hotspot is functionally essential for the biochemical and functional interaction between ARID1A and EZH2. The immune-defective phenotype of the ARID1A mutation may potentially explain the high susceptibility to chronic respiratory infection in patients with symptoms of Coffin-Siris syndrome who bear an ARID1A mutation (63). Thus, the antagonism of ARID1A and EZH2 may determine tumor cell IFN responsiveness, shape cancer immune phenotype, and potentially affect immunotherapy outcomes. We suggest that frequent intrinsic ARID1A mutations or low expression confer an unappreciated tumor immune evasion mechanism. Therefore, targeting BAF complex mutation-associated pathways in combination with immunotherapy may be a novel approach to treating patients with BAF-mutated cancers.

Opposite to our demonstration that ARID1A mutations negatively shape tumor immune phenotype, T cell immunity, and clinical outcome across many types of human cancer, a recent report has shown that ARID1A mutations are associated with improved outcome in UCEC patients (44). We have successfully reproduced this survival analysis in this patient population. However, we failed to detect an immune signature difference between WT ARID1A and mutated ARID1A UCEC patients. In addition, similar to our data from murine tumors with ARID1A knockdown, Shen et al. have demonstrated a decrease in tumor $\mathrm{T}$ cell infiltration in mice bearing ID8 tumors with ARID1A deficiency (44). Surprisingly, they found that decreased $\mathrm{CD}^{+} \mathrm{T}$ cell tumor infiltration is associated with reduced ID8 tumor growth (44). Thus, we speculate that the impact of ARID1A mutations on cancer progression and/ or $\mathrm{T}$ cell immunity may be influenced by other confounding element(s). We have primarily evaluated this possibility in patients with UCEC. It is well known that UCEC includes highly mutated tumor subtypes (64). POLE and PIK3CA are frequently and concurrently mutated with ARID1A $(32,48)$. Patients with POLEmutant tumors have significantly improved survival $(32,48)$. Thus, we have reanalyzed the relationship between ARID1A mutations and UCEC patient outcome by excluding those whose tumors have POLE and PIK3CA mutations. As expected, ARID1A mutations were no longer positively associated with UCEC patient outcome. More importantly, when we reevaluated pan-cancer patients by excluding patients with UCEC or patients with POLE and/or PIK3CA mutations, we validated that ARID1A mutations or low ARID1A copy number remained negatively associated with clinical outcome. Therefore, we suggest that ARID1A positively affects tumor immunity and patient survival in the majority of cancer histologies, but is probably context dependent, since ARID1A does not appear to play an independent immunological role in UCEC patients or in patients with high POLE and PIK3CA mutations.

In summary, we have systematically demonstrated that ARID1A biochemically, functionally, and clinically shapes tumor immunity and therapeutic response to immunotherapy across many types of human cancer. Targeting ARID1A-associated pathways may be an anticancer therapeutic approach.

\section{Methods}

Detailed information is provided in the Supplemental Methods.

Data access. ATAC-Seq, ARID1A, and GSK126 RNA-Seq data of OVCA429 cells were deposited into the National Center for Biotechnology Information's Gene Expression Omnibus database under GSE131918 (http://www.ncbi.nlm.nih.gov/geo).

Study approval. This study was approved by the Institutional Animal Care \& Use Committee of the University of Michigan. 


\section{Author contributions}

JL, Weichao Wang, RZ, AMC, KC, YD, IK, DH, and WZ conceived the project and designed the experiments. JL and Weichao Wang performed most of the experiments with help from YZ, MC, MT, LZ, BB, XJ, SW, HL, and JZ. LV, YZ, JL, and MC performed bioinformatics analysis. JL, Weimin Wang, YZ, MC, LZ, JG, MDG, TM, Weichao Wang, WL, TC, and IK contributed to investigation. DH, TC, and KC contributed to the sequencing data sets. JL, Weichao Wang, and WZ wrote the original draft and reviewed and edited the manuscript. AMC and WZ acquired funding. IK and WZ supervised the study.

\section{Acknowledgments}

We thank Lin Zhang for intellectual input and discussion, Cigall Kadoch for the ARID1A CRISPR plasmid, and Yikan Wang,
Ali Bashashati, Xuhong Cao, and Rui Wang for their technical assistance for this work. This work was supported in part by the Ovarian Cancer Research Alliance Ann and Sol Schreiber Mentored Investigator Award (to JL), T32 training grant (to JL) from the National Institute of Allergy and Infectious Diseases (AI007413), and research funding from NIH/NCI R01 grants (to WZ) (CA217648, CA123088, CA099985, CA193136, and CA152470), and the NIH through the University of Michigan Rogel Cancer Center Grant (CA46592).

Address correspondence to: Weiping Zou, University of Michigan School of Medicine, BSRB, 109 Zina Pitcher Place, Ann Arbor, Michigan 48109-0669, USA. Phone: 734.615.5554; Email: wzou@ med.umich.edu.
1. Zhang L, et al. Intratumoral T cells, recurrence, and survival in epithelial ovarian cancer. $N$ Engl J Med. 2003;348(3):203-213.

2. Galon J, et al. Type, density, and location of immune cells within human colorectal tumors predict clinical outcome. Science. 2006;313(5795):1960-1964.

3. Kryczek I, et al. Phenotype, distribution, generation, and functional and clinical relevance of Th17 cells in the human tumor environments. Blood. 2009;114(6):1141-1149.

4. Shin DS, et al. Primary resistance to PD-1 blockade mediated by JAK1/2 mutations. Cancer Discov. 2017;7(2):188-201.

5. Zou W, Wolchok JD, Chen L. PD-L1 (B7-H1) and PD-1 pathway blockade for cancer therapy: mechanisms, response biomarkers, and combinations. Sci Transl Med. 2016;8(328):328rv4.

6. Hugo W, et al. Genomic and transcriptomic features of response to anti-PD-1 therapy in metastatic melanoma. Cell. 2016;165(1):35-44.

7. Patel SJ, et al. Identification of essential genes for cancer immunotherapy. Nature. 2017;548(7669):537-542.

8. Park S, et al. The therapeutic effect of anti-HER2/ neu antibody depends on both innate and adaptive immunity. Cancer Cell. 2010;18(2):160-170.

9. Liu X, et al. CD47 blockade triggers T cellmediated destruction of immunogenic tumors. Nat Med. 2015;21(10):1209-1215.

10. Gajewski TF, Schreiber H, Fu YX. Innate and adaptive immune cells in the tumor microenvironment. Nat Immunol. 2013;14(10):1014-1022.

11. Kortlever RM, et al. Myc Cooperates with Ras by Programming Inflammation and Immune Suppression. Cell. 2017;171(6):1301-1315.e14.

12. Casey SC, et al. MYC regulates the antitumor immune response through CD47 and PD-L1. Science. 2016;352(6282):227-231.

13. Pylayeva-Gupta Y, Lee KE, Hajdu CH, Miller G, Bar-Sagi D. Oncogenic Kras-induced GM-CSF production promotes the development of pancreatic neoplasia. Cancer Cell. 2012;21(6):836-847.

14. Wörmann SM, et al. Loss of P53 function activates JAK2-STAT3 signaling to promote pancreatic tumor growth, stroma modification, and gemcitabine resistance in mice and is associated with patient survival. Gastroenterology. 2016;151(1):180-193.e12.
15. Cooks T, et al. Mutant p53 prolongs NF- $\kappa B$ activation and promotes chronic inflammation and inflammation-associated colorectal cancer. Cancer Cell. 2013;23(5):634-646.

16. Wiegand KC, et al. ARID1A mutations in endometriosis-associated ovarian carcinomas. N Engl JMed. 2010;363(16):1532-1543.

17. Jones $\mathrm{S}$, et al. Frequent mutations of chromatin remodeling gene ARID1A in ovarian clear cell carcinoma. Science. 2010;330(6001):228-231.

18. Wang K, et al. Exome sequencing identifies frequent mutation of ARID1A in molecular subtypes of gastric cancer. Nat Genet. 2011;43(12):1219-1223.

19. Kadoch C, et al. Proteomic and bioinformatic analysis of mammalian SWI/SNF complexes identifies extensive roles in human malignancy. Nat Genet. 2013;45(6):592-601.

20. Workman JL, Kingston RE. Alteration of nucleosome structure as a mechanism of transcriptional regulation. Annu Rev Biochem. 1998;67:545-579.

21. Kadoch C, Crabtree GR. Mammalian SWI/SNF chromatin remodeling complexes and cancer: mechanistic insights gained from human genomics. Sci Adv. 2015;1(5):e1500447.

22. Lewis EB. A gene complex controlling segmentation in Drosophila. Nature. 1978;276(5688):565-570.

23. Struhl G. A gene product required for correct initiation of segmental determination in Drosophila. Nature. 1981;293(5827):36-41.

24. Kennison JA, Tamkun JW. Dosage-dependent modifiers of polycomb and antennapedia mutations in Drosophila. Proc Natl Acad Sci U S A. 1988;85(21):8136-8140.

25. Wilson BG, et al. Epigenetic antagonism between polycomb and SWI/SNF complexes during oncogenic transformation. Cancer Cell. 2010;18(4):316-328

26. Bitler BG, et al. Synthetic lethality by targeting EZH2 methyltransferase activity in ARID1Amutated cancers. Nat Med. 2015;21(3):231-238.

27. Nagarsheth N, et al. PRC2 epigenetically silences Th1-type chemokines to suppress effector T-cell trafficking in colon cancer. Cancer Res. 2016;76(2):275-282.

28. Peng D, et al. Epigenetic silencing of TH1-type chemokines shapes tumour immunity and immunotherapy. Nature. 2015;527(7577):249-253.
29. Robinson DR, et al. Integrative clinical genomics of metastatic cancer. Nature. 2017;548(7667):297-303.

30. Wu R, et al. Mouse model of human ovarian endometrioid adenocarcinoma based on somatic defects in the Wnt/ $\beta$-catenin and PI3K/Pten signaling pathways. Cancer Cell. 2007;11(4):321-333.

31. Riaz N, et al. Tumor and microenvironment evolution during immunotherapy with Nivolumab. Cell. 2017;171(4):934-949.e16.

32. Cancer Genome Atlas Research Network, et al. The Cancer Genome Atlas Pan-Cancer analysis project. Nat Genet. 2013;45(10):1113-1120.

33. Wang W, et al. Effector T cells abrogate stromamediated chemoresistance in ovarian cancer. Cell. 2016;165(5):1092-1105.

34. Kryczek I, et al. IL-22(+)CD4(+) T cells promote colorectal cancer stemness via STAT3 transcription factor activation and induction of the methyltransferase DOT1L. Immunity. 2014;40(5):772-784.

35. Mathur R, et al. ARID1A loss impairs enhancermediated gene regulation and drives colon cancer in mice. Nat Genet. 2017;49(2):296-302.

36. Kelso TWR, Porter DK, Amaral ML, Shokhirev MN, Benner C, Hargreaves DC. Chromatin accessibility underlies synthetic lethality of SWI/ SNF subunits in ARID1A-mutant cancers. Elife. 2017;6:e30506.

37. Corces MR, et al. The chromatin accessibility landscape of primary human cancers. Science. 2018;362(6413):eaav1898.

38. Chang MT, et al. Identifying recurrent mutations in cancer reveals widespread lineage diversity and mutational specificity. Nat Biotechnol. 2016;34(2):155-163.

39. McCabe MT, et al. EZH2 inhibition as a therapeutic strategy for lymphoma with EZH2-activating mutations. Nature. 2012;492(7427):108-112.

40. Yadav M, et al. Predicting immunogenic tumour mutations by combining mass spectrometry and exome sequencing. Nature. 2014;515(7528):572-576.

41. Rizvi NA, et al. Cancer immunology. Mutational landscape determines sensitivity to PD-1 blockade in non-small cell lung cancer. Science. 2015;348(6230):124-128

42. Van Allen EM, et al. Genomic correlates of response to CTLA- 4 blockade in metastatic mel- 
anoma. Science. 2015;350(6257):207-211.

43. Roh W, et al. Integrated molecular analysis of tumor biopsies on sequential CTLA-4 and PD- 1 blockade reveals markers of response and resistance. Sci Transl Med. 2017;9(379):eaah3560.

44. Shen J, et al. ARID1A deficiency promotes mutability and potentiates therapeutic antitumor immunity unleashed by immune checkpoint blockade. Nat Med. 2018;24(5):556-562.

45. Cancer Genome Atlas Research Network. Integrated genomic analyses of ovarian carcinoma. Nature. 2011;474(7353):609-615.

46. Zehir A, et al. Mutational landscape of metastatic cancer revealed from prospective clinical sequencing of 10,000 patients. Nat Med. 2017;23(6):703-713.

47. Liu J, et al. An Integrated TCGA pan-cancer clinical data resource to drive high-quality survival outcome analytics. Cell. 2018;173(2):400-416.e11.

48. Espinosa I, Lee CH, D’Angelo E, Palacios J, Prat J. Undifferentiated and dedifferentiated endometrial carcinomas with POLE exonuclease domain mutations have a favorable prognosis. Am J Surg Pathol. 2017;41(8):1121-1128.

49. Kuo KT, et al. Frequent activating mutations of PIK3CA in ovarian clear cell carcinoma. Am J Pathol. 2009;174(5):1597-1601.

50. Shain AH, Pollack JR. The spectrum of SWI/SNF mutations, ubiquitous in human cancers. PLoS One. 2013;8(1):e55119.

51. Giannakis M, et al. Genomic correlates of immune-cell infiltrates in colorectal carcinoma. Cell Rep. 2016;15(4):857-865.

52. Cajuso T, et al. Exome sequencing reveals frequent inactivating mutations in ARID1A, ARID1B, ARID2 and ARID4A in microsatellite unstable colorectal cancer. Int J Cancer. 2014;135(3):611-623.

53. Schumacher TN, Schreiber RD. Neoantigens in cancer immunotherapy. Science. 2015;348(6230):69-74.

54. Curiel TJ, et al. Blockade of B7-H1 improves myeloid dendritic cell-mediated antitumor immunity. Nat Med. 2003;9(5):562-567.

55. Curiel TJ, et al. Specific recruitment of regulatory $\mathrm{T}$ cells in ovarian carcinoma fosters immune privilege and predicts reduced survival. Nat Med. 2004;10(9):942-949.

56. Cui TX, et al. Myeloid-derived suppressor cells enhance stemness of cancer cells by inducing microRNA101 and suppressing the corepressor CtBP2. Immunity. 2013;39(3):611-621.

57. Spranger S, Bao R, Gajewski TF. Melanomaintrinsic $\beta$-catenin signalling prevents anti-tumour immunity. Nature. 2015;523(7559):231-235.

58. Li W, et al. Aerobic glycolysis controls myeloid-derived suppressor cells and tumor immunity via a specific CEBPB isoform in triple-negative breast cancer. Cell Metab. 2018;28(1):87-103.e6.

59. Lin H, et al. Host expression of PD-L1 determines efficacy of PD-L1 pathway blockademediated tumor regression. J Clin Invest. 2018;128(2):805-815.

60. Lemon B, Inouye C, King DS, Tjian R. Selectivity of chromatin-remodelling cofactors for ligand-activated transcription. Nature. 2001;414(6866):924-928.

61. Pan D, et al. A major chromatin regulator determines resistance of tumor cells to T cell-mediated killing. Science. 2018;359(6377):770-775.

62. Miao D, et al. Genomic correlates of response to immune checkpoint therapies in clear cell renal cell carcinoma. Science. 2018;359(6377):801-806.

63. Wieczorek D, et al. A comprehensive molecular study on Coffin-Siris and Nicolaides-Baraitser syndromes identifies a broad molecular and clinical spectrum converging on altered chromatin remodeling. Hum Mol Genet. 2013;22(25):5121-5135.

64. Kandoth C, et al. Mutational landscape and significance across 12 major cancer types. Nature. 2013;502(7471):333-339.

65. Dennis G, et al. DAVID: database for annotation, visualization, and integrated discovery. Genome Biol. 2003;4(5):P3. 